\title{
Bipower-type estimation in a noisy diffusion setting*
}

\author{
Mark Podolskij ${ }^{\dagger}$ \\ University of Aarhus and CREATES \\ Mathias Vetter ${ }^{\ddagger}$ \\ Ruhr-Universität Bochum
}

May 23, 2008

\begin{abstract}
We consider a new class of estimators for volatility functionals in the setting of frequently observed Itō diffusions which are disturbed by i.i.d. noise. These statistics extend the approach of pre-averaging as a general method for the estimation of the integrated volatility in the presence of microstructure noise and are closely related to the original concept of bipower variation in the no-noise case. We show that this approach provides efficient estimators for a large class of integrated powers of volatility and prove the associated (stable) central limit theorems. In a more general Itō semimartingale framework this method can be used to define both estimators for the entire quadratic variation of the underlying process and jump-robust estimators which are consistent for various functionals of volatility. As a by-product we obtain a simple test for the presence of jumps in the underlying semimartingale.

Keywords: Bipower Variation; Central Limit Theorem; High-Frequency Data; Microstructure Noise; Quadratic Variation; Semimartingale Theory; Test for Jumps.
\end{abstract}

${ }^{*}$ The first author acknowledges financial support from CREATES funded by the Danish National Research Foundation. The work of the second author was supported by the Deutsche Forschungsgemeinschaft (SFB 475, Komplexitätsreduktion in multivariaten Datenstrukturen).

${ }^{\dagger}$ CREATES, School of Economics and Management, University of Aarhus, Building 1322, DK-8000 Aarhus C, Denmark. E-mail: mpodolskij@creates.au.dk.

${ }^{\ddagger}$ Ruhr-Universität Bochum, Fakultät für Mathematik, D-44780 Bochum, Germany. Tel: +49-2343223283, Fax: +49-234-3214559. E-mail: mathias.vetter@rub.de 


\section{Introduction}

The last years have seen a rapidly growing literature on the estimation of volatility in case of high frequency data. Starting from the representation of (log) price processes as Itō diffusions, which are widely accepted as a reasonable model for stock or currency prices, empirical research suggests that the true observations are contaminated by microstructure noise, which collects deviations from the true and the observed prices that are due to bid-ask spreads or round-off errors, among others. These effects seem to have a huge impact on the performance of the classical estimators in the pure diffusion case, which explains the need for a general theory of the treatment of microstructure noise.

Throughout this paper we will focus on a general nonparametric setting, thus the underlying diffusion process is characterised by the equation

$$
X_{t}=X_{0}+\int_{0}^{t} a_{s} d s+\int_{0}^{t} \sigma_{s} d W_{s},
$$

whereas $\left(a_{s}\right)$ denotes a predictable locally bounded drift and $\left(\sigma_{s}\right)$ a càdlàg volatility process. Since we are dealing with high frequeny data, we assume the process to live on a fixed time interval, [0,1] say. A typical quantity of interest is the integrated volatility $\int_{0}^{1} \sigma_{s}^{2} d s$, for which the realised variance $\sum_{i=1}^{n}\left|\Delta_{i}^{n} X\right|^{2}$ is a natural estimator in the case of non-noisy observations. See for example Andersen et al. [3] or Barndorff-Nielsen and Shephard [6]. Microstructure noise has commonly been modelled as an additive error, which fulfills some moment conditions and behaves essentially like a white noise process. However, a more general setting is possible and was discussed in Jacod et al. [14].

It was shown in Zhang et al. [22] that the realised variance becomes inconsistent when dealing with microstructure noise, which started the search for new methods to solve the problem of volalitity estimation in this context. Up to now, there exist three approaches to this question. Zhang et al. [22] and Zhang [21] used linear combinations of increments at different time lags to define a subsampling estimator, whereas Barndorff-Nielsen et al. [5] proposed a kernel based estimator, which essentially consists of a weighted sum of autocovariances. The method of pre-averaging over small intervals was introduced in Podolskij and Vetter [18] and to a first extent generalised in Jacod et al. [14]. Each approach provides consistent 
estimators and achieves the optimal rate of convergence of $n^{-\frac{1}{4}}$ in a stable limit theorem.

In this paper we propose a class of bipower-type estimators which are preaveraged analogues of the realised bipower variation

$$
B V(X, l, r)^{n}=n^{\frac{r+l}{2}-1} \sum_{i=1}^{n-1}\left|\Delta_{i}^{n} X\right|^{l}\left|\Delta_{i+1}^{n} X\right|^{r},
$$

which was considered in Barndorff-Nielsen et al. [4]. We prove stochastic convergence and state joint central limit theorems of these bipower-type statistics, both for any choice of non-negative powers $l$ and $r$. As in earlier work on pre-averaged estimators, simple modifications of such bipower-type statistics turn out to be consistent for a large class of integrated powers of volatility.

In contrast to the original concept of modulated bipower variation as defined in Podolskij and Vetter [18] this new method also serves as a powerful tool to draw inference about the underlying price process even in the case, where it is defined as a realisation of an Itō semimartingale

$$
X_{t}=X_{0}+B+X^{c}+\kappa \star(\mu-\nu)+\kappa^{\prime} \star \mu,
$$

which does not necessarily have continuous paths as in (1.1), but allows for jumps as well. A precise definition of the processes involved will be given later.

In this rather general setting we construct both a consistent estimator for the entire quadratic variation of $X$ and jump-robust estimators which are consistent for the integrated powers of volatility. In a similar way as in the no-noise case (see Barndorff-Nielsen and Shephard [7] or Ait-Sahalia and Jacod [1], among others) we are then able to solve the problem, how to test for jumps of the process $X$ in the presence of microstructure noise. Based on the estimator for the quadratic variation of $X$ and the robust one for the integrated volatility we construct two test statistics, which are given by differentiable functions of two bipower-type statistics with different powers $l$ and $r$. By means of a joint central limit theorem we obtain two simple tests for the presence of jumps, both under the null hypothesis of no jumps.

This paper is organised as follows: In Section 2 we state the assumptions and define the class of bipower-type statistics. Section 3 is devoted to the asymptotic 
results, whereas Section 4 deals with their applications in a test for jumps. All proofs are given in Section 5.

\section{Assumptions and definitions}

We assume that the underlying continuous process $X=\left(X_{t}\right)_{t}$ is a diffusion process as given in (1.1), which is defined on an appropriate filtered probability space $\left(\Omega^{(0)}, \mathcal{F}^{(0)},\left(\mathcal{F}_{t}^{(0)}\right)_{t \in[0,1]}, P^{(0)}\right)$. As noted before, we assume further that the process lives on the time interval $[0,1]$.

Since we are dealing with microstructure noise we have to define a second process $Z=\left(Z_{t}\right)_{t}$, which is somehow connected to the underlying Itō semimartingale $X$. We restrict ourselves to the case of i.i.d. noise, which means that the observed data are given by

$$
Z_{t_{n, i}}=X_{t_{n, i}}+U_{t_{n, i}}
$$

at each observation time $t_{n, i}$, where $U_{t}, t \in[0,1]$, is an i.i.d. noise process independent of $X$ with

$$
E\left[U_{t}\right]=0 \quad \text { and } \quad E\left[U_{t}^{2}\right]=\omega^{2} .
$$

Furthermore, we assume that for each $n$ the observation times are given by $t_{n, i}=$ $\frac{i}{n}, 0 \leq i \leq n$.

In order to make both $X$ and $Z$ measurable with respect to the same kind of filtration, we have to define a new probability space $\left(\Omega, \mathcal{F},\left(\mathcal{F}_{t}\right)_{t}, P\right)$, which accommodates both processes. To this end, we assume similarly to the setting in Jacod et al. [14] that one has a second space $\left(\Omega^{(1)}, \mathcal{F}^{(1)},\left(\mathcal{F}_{t}^{(1)}\right)_{t \in[0,1]}, P^{(1)}\right)$, where $\Omega^{(1)}$ denotes $\mathbb{R}^{[0,1]}$ and $\mathcal{F}^{(1)}$ the product Borel- $\sigma$-field on $\Omega^{(1)}$. Furthermore, for any $t \in[0,1]$ we define $Q_{t}\left(\omega^{(0)}, d z\right)$ to be the probability measure, which corresponds to the transition from $X_{t}\left(\omega^{(0)}\right)$ to the observed process $Z_{t}$. In the case of i.i.d. noise, this transition kernel is rather simple, since we have

$$
Q_{t}\left(\omega^{(0)}, d z\right)=g\left(z-X_{t}\left(\omega^{(0)}\right)\right) d z
$$

whenever $U$ has a density $g$. We define at last $P^{(1)}\left(\omega^{(0)}, d \omega^{(1)}\right)$ to be the product $\otimes_{t \in[0,1]} Q_{t}\left(\omega^{(0)}, \cdot\right)$. By construction, $\left(Z_{t}\right)_{t}$ can be regarded as the canonical process on $\left(\Omega^{(1)}, \mathcal{F}^{(1)}, P^{(1)}\right)$ with the natural filtration given by $\mathcal{F}_{t}^{(1)}=\sigma\left(Z_{s} ; s \leq t\right)$. The filtered probability space $\left(\Omega, \mathcal{F},\left(\mathcal{F}_{t}\right)_{t \in[0,1]}, P\right)$ is then defined as 


$$
\left.\begin{array}{l}
\Omega=\Omega^{(0)} \times \Omega^{(1)}, \quad \mathcal{F}=\mathcal{F}^{(0)} \times \mathcal{F}^{(1)}, \quad \mathcal{F}_{t}=\bigcap_{s>t} \mathcal{F}_{s}^{(0)} \times \mathcal{F}_{s}^{(1)}, \\
P\left(d \omega^{(0)}, d \omega^{(1)}\right)=P^{(0)}\left(d \omega^{(0)}\right) P^{(1)}\left(\omega^{(0)}, d \omega^{(1)}\right) .
\end{array}\right\}
$$

Remark 1 Note that this setting refers in view of Jacod et al. [14] only to a special case of a noisy observation scheme. However, even in the more general case presented therein the process $Z$ exhibits a decomposition of the form

$$
Z_{t}=X_{t}+h_{t} U_{t}
$$

where $h_{t}$ is $\mathcal{F}^{(0)}$-measurable and conditionally on $\mathcal{F}^{(0)}$ the $U_{t}$ have mean zero, unit variance and $\left(U_{t}, U_{s}\right)$ are mutually independent for all $t \neq s$. This representation as well as the results from Jacod et al. [14] indicate that main results from this paper may be derived in the general setting as well.

Before we are able to define the class of bipower-type statistics $B T(l, r)^{n}$ we have to introduce some further items and notations. First, we choose a sequence $k_{n}$ of integers, for which a positive number $\theta$ satisfying

$$
\frac{k_{n}}{\sqrt{n}}=\theta+o\left(n^{-\frac{1}{4}}\right)
$$

exists, and a nonzero real-valued function $g: \mathbb{R} \rightarrow \mathbb{R}$, which fulfills the following conditions:

(i) $g$ vanishes outside of $(0,1)$

(ii) $g$ is continuous and piecewise $C^{1}$

(iii) Its derivative $g^{\prime}$ is piecewise Lipschitz.

We associate with $g$ the following real valued numbers:

$$
\left.\begin{array}{l}
g_{i}^{n}=g\left(\frac{i}{k_{n}}\right), \quad \psi_{2}=\int_{0}^{1}(g(s))^{2} d s, \quad \psi_{2}^{n}=\frac{1}{k_{n}} \sum_{i=1}^{k_{n}-1}\left(g_{i}^{n}\right)^{2}, \\
\psi_{1}=\int_{0}^{1}\left(g^{\prime}(s)\right)^{2} d s, \quad \psi_{1}^{n}=k_{n} \sum_{i=0}^{k_{n}-1}\left(g_{i+1}^{n}-g_{i}^{n}\right)^{2} .
\end{array}\right\}
$$

Furthermore, for any process $V=\left(V_{t}\right)$ we define the random variables

$$
\left.\begin{array}{l}
V_{i}^{n}=V_{\frac{i}{n}}, \quad \Delta_{i}^{n} V=V_{i}^{n}-V_{i-1}^{n}, \\
\overline{\Delta_{i}^{n} V}=V_{i+k_{n}}^{n}-V_{i}^{n}, \quad \bar{V}_{i}^{n}=\sum_{j=1}^{k_{n}} g_{j}^{n} \Delta_{i+j}^{n} V .
\end{array}\right\}
$$


Note that $\bar{V}_{i}^{n}$ can be represented as

$$
\bar{V}_{i}^{n}=\int_{\frac{i}{n}}^{\frac{i+k_{n}}{n}} g_{n}\left(s-\frac{i}{n}\right) d V_{s} \quad \text { with } \quad g_{n}(s)=\sum_{j=1}^{k_{n}} g_{j}^{n} 1_{\left(\frac{j-1}{n}, \frac{j}{n}\right]}(s) .
$$

For any process $V$ and two arbitrary positive real numbers $l$ and $r$ the bipowertype statistic $B T(V, l, r)^{n}$ is then defined as

$$
B T(V, l, r)^{n}=n^{\frac{l+r}{4}-1} \sum_{i=0}^{n-2 k_{n}+1}\left|\bar{V}_{i}^{n}\right|^{l}\left|\bar{V}_{i+k_{n}}^{n}\right|^{r} .
$$

If we simply write $B T(l, r)^{n}$, we assume that we define this statistic with respect to $Z$. At least one example for a bipower-type estimator has already been studied, since in Jacod et al. [14] a slight modification of $B T(2,0)^{n}$ was shown to be a consistent estimator of the integrated volatility of the underlying process $X$.

This class of estimators generalises the approach of modulated bipower variation as proposed in Podolskij and Vetter [18] in a twofold manner:

First, instead of using the simple kernel function

$$
g(x)=(x \wedge(1-x))^{+}
$$

we allow for different types of weights on the increments $\Delta_{i}^{n} Z$. Similarly to Podolskij and Vetter [18] the choice of $k_{n}$ ensures that the stochastic orders of $\bar{X}_{i}^{n}$ and $\bar{U}_{i}^{n}$ are balanced, which explains why characteristics of $X$ and $U$ will both be present in the stochastic limit and the central limit theorem.

Second, we do not only sum up such statistics $\bar{Z}_{i}^{n}$, which are defined over nonoverlapping intervals of length $\frac{k_{n}}{n}$, but use all available statistics up to time $1-\frac{2 k_{n}}{n}$. This change does not affect the behaviour in the stochastic limit, but certainly it increases the estimator's efficiency. Most important, however: When the underlying process allows for jumps as in (1.3), we need estimators that give equal weight to any increment of lag $\frac{1}{n}$ (apart from increments on the boundary of $[0,1]$ ) in order to draw inference about the quadratic variation of the jump part.

A third generalisation towards multipower-type statistics, which can be defined as sums of products of more than two adjacent pre-averaged statistics, will not be derived in this paper. Inferences about these estimators can be obtained by extensions of the following results in a straightforward way. 
We finish this section with some words on the representation of a semimartingale $X$ as defined in (1.3). Its representation is defined as in Jacod and Shiryaev [16]: $\mu$ denotes a jump measure and $\nu$ its predictable time compensator, whereas integrals of optional functions $V$ with respect to a random measure $\mu$ are denoted by $V \star \mu$. $\kappa$ is assumed to be a fixed truncation function, which is continuous, has compact support and coincides with the identity on a neighbourhood of zero. $\kappa^{\prime}$ is defined via $\kappa^{\prime}(x)=x-\kappa(x)$. Moreover, $X^{c}$ denotes the continuous martingale part and $(B, C, \nu)$ with $C=<X^{c}, X^{c}>$ are the predictable characteristics of $X$.

\section{Asymptotic theory}

In this section we study the asymptotic behaviour of the class of bipower-type estimators $B T(l, r)^{n}, l, r \geq 0$. In the pure diffusion case we obtain stochastic convergence for each choice of $l$ and $r$ under mild assumptions, since apart from a moment condition on the noise process $U$ no further assumptions on $Z$ are needed. In order to prove a central limit theorem we have to modify the setting slighty, but are still able to derive results for a large class of volatility processes. In the semimartingale framework we will restrict ourselves to less general choices of $l$ and $r$.

\subsection{Consistency}

We start with the statement of the stochastic limit in case $X$ is a continuous Itō diffusion as defined in (1.1).

Theorem 1 Assume that $E|U|^{2(l+r)+\epsilon}<\infty$ for some $\epsilon>0$ and let $\mu_{r}$ denote the $r$-th absolute moment of a standard normal distribution. Then the convergence in probability

$$
B T(l, r)^{n} \stackrel{P}{\longrightarrow} B T(l, r)=\mu_{l} \mu_{r} \int_{0}^{1}\left(\theta \psi_{2} \sigma_{u}^{2}+\frac{1}{\theta} \psi_{1} \omega^{2}\right)^{\frac{l+r}{2}} d u
$$

holds.

The moment condition on $U$ is crucial to replace the moments of $\bar{U}_{i}^{n}$ by the corresponding moments of a standard normal distribution which only depend on $\omega^{2}$. 
Remark 2 Theorem 1 indicates that the class of bipower-type estimators is inconsistent for any integrated power of volatility. However, when $l+r$ is an even number, a modification of $B T(l, r)^{n}$ similar to the one in Podolskij and Vetter [18] turns out to be consistent. This can be illustrated as follows: Since

$$
\hat{\omega}^{2}=\frac{1}{2 n} \sum_{i=1}^{n}\left|\Delta_{i}^{n} Z\right|^{2}
$$

is a constistent estimator for $\omega^{2}$ (see e.g. Zhang et al. [22]), one obtains consistent estimators for integrated powers of volatility, as long as one is able to estimate and subtract the bias due to $\omega^{2}$ in the stochastic limit $B T(l, r)$. When $\frac{l+r}{2}$ is an integer, this is of course a simple application of the binomial theorem. The special case of $B T(2,0)^{n}$ has already been treated in Jacod et al. [14], where

$$
\hat{C}^{n}=\frac{1}{\theta \psi_{2}} B T(2,0)^{n}-\frac{\psi_{1}}{\theta^{2} \psi_{2}} \hat{\omega}^{2} \stackrel{P}{\longrightarrow} \int_{0}^{1} \sigma_{s}^{2} d s
$$

was introduced as an estimator of the integrated volatility.

However, if $X$ is supposed to be a semimartingale, Theorem 1 does not hold in general. Nevertheless, in the spirit of Jacod [12] it is possible to show the stochastic convergence of $B T(l, r)^{n}$ (or a rescaled version), where the limit depends both on the choice of $l$ and $r$ and on additional assumptions on the processes involved. We will investigate the cases which are important in order to derive estimators for the entire quadratic variation or parts thereof. Since we want to focus on Itō semimartingales only, we need an additional assumption on the characteristics of $X$, which ensures that its drift and its continuous martingale part are given by an Itō diffusion. Furthermore, a certain structure on the compensator $\nu$ is imposed.

(H): The characteristics $(B, C, \nu)$ of the semimartingale $X$ are as follows:

$$
B_{t}=\int_{0}^{t} a_{s} d s, \quad C_{t}=\int_{0}^{t} \sigma_{s}^{2} d s, \quad \nu(d t, d x)=d t F_{t}(d x),
$$

whereas the processes $\left(a_{s}\right)$ and $\left(F_{s}\left(\Phi_{2}\right)\right)$ are locally bounded and predictable. Here, $F_{s}(f)$ denotes the integral $\int f(x) F_{s}(d x)$ and

$$
\Phi_{r}(x)=1 \wedge|x|^{r},
$$

$r>0$. Moreover, $\left(\sigma_{s}\right)$ is assumed to be càdlàg. 
This condition is the same condition as in Jacod [12]. It implies that $X_{t}$ can be represented in the following way:

$$
\begin{aligned}
X_{t} & =X_{0}+\int_{0}^{t} a_{s} d s+\int_{0}^{t} \sigma_{s} d W_{s} \\
& +\int_{0}^{t} \int_{\mathbb{R}} \kappa \circ \delta(s, x)(\underline{\mu}-\underline{\nu})(d s, d x)+\int_{0}^{t} \int_{\mathbb{R}} \kappa^{\prime} \circ \delta(s, x) \underline{\mu}(d s, d x),
\end{aligned}
$$

where $\underline{\mu}$ is a Poisson random measure on $\mathbb{R}_{+} \times \mathbb{R}$ with its compensator $\underline{\nu}(d t, d x)=$ $d t \times d x . \delta$ is a function from $\Omega \times \mathbb{R}_{+} \times \mathbb{R}$ to $\mathbb{R}$, such that $F_{t}(\omega, d x)$ is the image of $d x$ under the mapping $x \mapsto \delta(\omega, s, x)$.

We can now state a result about the stochastic convergence of $B T(l, r)^{n}$ in the general semimartingale context.

Theorem 2 Assume that the underlying process $X$ is given by (1.3) and that both (H) and the conditions on $U$ from Theorem 1 are fulfilled. Then

(i)

$$
B T(2,0)^{n} \stackrel{P}{\longrightarrow} \int_{0}^{1} \theta \psi_{2} \sigma_{u}^{2} d u+\theta \psi_{2} \sum_{s \leq 1}\left|\Delta X_{s}\right|^{2}+\frac{1}{\theta} \psi_{1} \omega^{2} .
$$

(ii) If $l \vee r<2$ then $B T(l, r)^{n}$ is robust to jumps, i.e. it converges in probalility to $B T(l, r)$ as given in (3.1).

We see that this result provides us with simple estimates for the joint quadratic variation of the process $X$, but gives also robust estimators for the integrated volatility. For example, we may conclude that

$$
\frac{1}{\theta \psi_{2}} B T(2,0)^{n}-\frac{\psi_{1}}{\theta^{2} \psi_{2}} \hat{\omega}^{2}
$$

is a consistent estimator for

$$
[X, X]_{1}=\int_{0}^{1} \sigma_{s}^{2} d s+\sum_{s \leq 1}\left|\Delta X_{s}\right|^{2}
$$

which is the quadratic variation of the process $X$ at time 1 . Moreover, we have

$$
B T V^{n}=B T(2,0)^{n}-\mu_{1}^{-2} B T(1,1)^{n} \stackrel{P}{\longrightarrow} \theta \psi_{2} \sum_{s \leq 1}\left|\Delta X_{s}\right|^{2},
$$


since $\mu_{1}^{-2} B T(1,1)^{n}$ converges to the integrated volatility even in the presence of jumps. Thus $B T V^{n}$ (or a slight modification thereof) quantifies the part of the quadratic variation, which is due to jumps. Based on this statistic we will in the following derive feasible tests for the presence of jumps in the latent process $X$. A second test will be based on the ratio of $B T(2,0)^{n}$ and $B T(1,1)^{n}$.

Statistics like $B T V^{n}$ are somewhat similar to the ones obtained by applying the original concept of bipower variation, which serves as an alternative method for the estimation of the integrated volatility in the presence of jumps, when no market microstructure noise is present.

\subsection{Central limit theorems}

In this paragraph we present a central limit theorem for a normalised version of $B T(l, r)^{n}$, where for the first part of this section $X$ is given by (1.1), thus having continuous paths. As mentioned before, further assumptions on the process $Z$ are required. At first, we need two structural assumptions on the volatility process $\sigma$, which are already known to be required for the proof of the central limit theorem for bipower variation in the no-noise-case, but were also used to derive a central limit theorem for modulated bipower variation (see e.g. Barndorff-Nielsen et al. [4] or Podolskij and Vetter [18]).

(V): The process $\sigma$ satisfies the equation

$$
\sigma_{t}=\sigma_{0}+\int_{0}^{t} a_{s}^{\prime} d s+\int_{0}^{t} \sigma_{s}^{\prime} d W_{s}+\int_{0}^{t} v_{s}^{\prime} d V_{s} .
$$

Here $a^{\prime}, \sigma^{\prime}$ and $v^{\prime}$ are adapted càdlàg processes, with $a^{\prime}$ also being predictable and locally bounded, and $V$ is a second Brownian motion, independent of $W$.

$$
\left(\mathbf{V}^{\prime}\right): \quad \sigma^{2}>0
$$

Assumption (V) is fulfilled in many widely used financial models (see Black and Scholes [8], Vasicek [20], Cox et al. [10] or Chan et al. [9] among others), since whenever $X$ is a unique strong solution of a stochastic differential equation with a 
volatility function $\sigma_{t}=\sigma\left(t, X_{t}\right)$ being smooth enough, condition $(\mathrm{V})$ with $v_{s}^{\prime}=0$ holds as a simple consequence of Itō's formula.

The assumptions on the noise process $U$ are less restrictive than in Podolskij and Vetter [18], where it was assumed that $U$ follows a normal distribution.

(A): For the noise variables $U$ we have the following conditions:

(i) $U$ is distributed symmetrically around zero.

(ii) For any $0>a>-1$ we have $E\left[|U|^{a}\right]<\infty$.

(A'): Cramer's condition is fulfilled, that is $\lim \sup _{|t| \rightarrow \infty} \chi(t)<1$, where $\chi$ denotes the characteristic function of $U$.

The first condition is of fundamental importance, if at least one of the powers $l$ and $r$ is smaller than one. In this case the corresponding central limit theorem for the classical bipower variation relies on the fact that the normal distribution satisfies both properties from (A). We will see later that for our purposes one has to proceed in a similar way, which explains this additional assumption on the noise process. (A') will be used in order to remove the intrinsic bias in the pre-averaged statistic $\left|\bar{Z}_{i}^{n}\right|^{l}$, when the power $l$ is not an even number. Typically we replace the moments of $\left|n^{\frac{1}{4}} \bar{U}_{i}^{n}\right|^{l}$ by the corresponding moments of a normal distribution, but a priori we have no information about the size of the error due to this replacement. In order to show that this error becomes sufficiently small, we will use an expansion of Edgeworth-type, for which (A') is a standard assumption. As in the previous section, we need an additional moment condition on $U$ as well, depending on the choice of $l$ and $r$.

All central limit theorems stated below will make use of the concept of stable convergence of random variables. Let us shortly recall the definition. A sequence of random variables $G_{n}$ is said to converge stably in law with limit $G$ (throughout this paper we write $G_{n} \stackrel{\mathcal{D}_{s t}}{\longrightarrow} G$ ), defined on an appropriate extension $\left(\Omega^{\prime}, \mathcal{F}^{\prime}, P^{\prime}\right)$ of the 
original probability space $(\Omega, \mathcal{F}, P)$, if and only if for any $\mathcal{F}$-measurable and bounded random variable $H$ and any bounded and continuous function $f$ the convergence

$$
\lim _{n \rightarrow \infty} E\left[H f\left(G_{n}\right)\right]=E[H f(G)]
$$

holds. This is obviously a slightly stronger mode of convergence than convergence in law (see Renyi [19] or Aldous and Eagleson [2] for more details on stable convergence).

Since we want to use $B T V^{n}$ as defined in (3.6) to establish a test for the presence of jumps in the underlying semimartingale, we state a central limit theorem for 2-dimensional arrays of bipower-type statistics. Therefore, we fix non-negative numbers $l_{1}, r_{1}, l_{2}, r_{2}$ and set

$$
\begin{aligned}
& \xi_{n}^{1}=B T\left(l_{1}, r_{1}\right)^{n}-B T\left(l_{1}, r_{1}\right), \\
& \xi_{n}^{2}=B T\left(l_{2}, r_{2}\right)^{n}-B T\left(l_{2}, r_{2}\right), \\
& \xi_{n}=\left(\xi_{n}^{1}, \xi_{n}^{2}\right) .
\end{aligned}
$$

Before we proceed with the central limit theorem for $\xi_{n}$, we have to introduce some further notation. We define

$$
h_{i j}(x, y, z)=\operatorname{Cov}\left(\left|H_{1}\right|^{l_{i}}\left|H_{2}\right|^{r_{i}},\left|H_{3}\right|^{l_{j}}\left|H_{4}\right|^{r_{j}}\right)
$$

where $x$ is a real number, $y$ and $z$ are a two- and four-dimensional vector, respectively, and $\left(H_{1}, \ldots, H_{4}\right)$ follows a normal distribution with

(i) $E\left[H_{l}\right]=0$ and $E\left[\left|H_{l}\right|^{2}\right]=y_{1} x^{2}+y_{2} \omega^{2}$.

(ii) $H_{1} \perp H_{2}, H_{1} \perp H_{4}$ and $H_{3} \perp H_{4}$.

$$
\operatorname{Cov}\left(H_{1}, H_{3}\right)=\operatorname{Cov}\left(H_{2}, H_{4}\right)=z_{1} x^{2}+z_{2} \omega^{2}
$$

and

$$
\operatorname{Cov}\left(H_{2}, H_{3}\right)=z_{3} x^{2}+z_{4} \omega^{2}
$$

Each $h_{i j}$ can in principle be computed, but the calculations become rather complicated, except for special cases. 
Moreover, we set $t=\left(\theta \psi_{2}, \frac{1}{\theta} \psi_{1}\right)$ and define the functions

$$
\begin{aligned}
& f_{1}(s)=\theta \int_{0}^{1-s} g(r) g(r+s) d r, \quad f_{2}(s)=\frac{1}{\theta} \int_{0}^{1-s} g^{\prime}(r) g^{\prime}(r+s) d r, \\
& f_{3}(s)=\theta \int_{0}^{2-s} g(r) g(r+s-1) d r, \quad f_{4}(s)=\frac{1}{\theta} \int_{0}^{2-s} g^{\prime}(r) g^{\prime}(r+s-1) d r
\end{aligned}
$$

for $s \in[0,2]$. Note that both $f_{1}$ and $f_{2}$ are 0 for $s \in[1,2]$, according to the assumptions on $g$.

The conditional variance in the following limit theorem depends on the functions $h_{i j}$ introduced above and will therefore not be computed explicitly. Nevertheless, we will explain afterwards, how it can be estimated consistently. This is sufficient to derive feasible central limit theorems.

Theorem 3 Let $l_{1}, r_{1}, l_{2}$ and $r_{2}$ be four positive real numbers and let $X$ be given by (1.1). We further assume (V) and (A), and impose additionally that $U$ fulfills $E\left[|U|^{s+\epsilon}\right]<\infty$ for some $s \geq\left(3 \wedge 2\left(r_{1}+l_{1}\right) \wedge 2\left(r_{2}+l_{2}\right)\right)$ and some $\epsilon>0$. If any $l_{i}$ or $r_{i}$ is in $(0,1]$, we postulate ( $\left.V^{\prime}\right)$ as well, otherwise either ( $\left.V^{\prime}\right)$ or $\left(A^{\prime}\right)$.

Then

$$
n^{\frac{1}{4}} \xi_{n} \stackrel{\mathcal{D}_{s t}}{\longrightarrow} V\left(l_{1}, r_{1}, l_{2}, r_{2}\right)
$$

where the limiting process is given by

$$
V\left(l_{1}, r_{1}, l_{2}, r_{2}\right)=\int_{0}^{1} v_{l_{1}, r_{1}, l_{2}, r_{2}}\left(\sigma_{u}\right) d W_{u}^{\prime}
$$

Here $W^{\prime}$ denotes a 2-dimensional standard Brownian motion, which is defined on an extension of the filtered probability space $\left(\Omega, \mathcal{F},\left(\mathcal{F}_{t}\right)_{t}, P\right)$ and is independent of the $\sigma$-field $\mathcal{F}$. The conditional variance of the limiting process is given by

$$
\int_{0}^{1} v_{l_{1}, r_{1}, l_{2}, r_{2}}^{t} v_{l_{1}, r_{1}, l_{2}, r_{2}}\left(\sigma_{u}\right) d u=\int_{0}^{1}\left(\begin{array}{ll}
w_{11}^{l_{1}, r_{1}, l_{2}, r_{2}} & w_{12}^{l_{1}, r_{1}, l_{2}, r_{2}} \\
w_{12}^{l_{1}, r_{1}, l_{2}, r_{2}} & w_{22}^{l_{1}, r_{1}, l_{2}, r_{2}}
\end{array}\right)\left(\sigma_{u}\right) d u
$$

where

$$
w_{i j}^{l_{1}, r_{1}, l_{2}, r_{2}}\left(\sigma_{u}\right)=2 \theta \int_{0}^{2} h_{i j}\left(\sigma_{u}, t, f(s)\right) d s
$$

In the following we will drop the arguments indicating the dependence of $v$ and $w_{i j}$ on the choice of $l_{1}, r_{1}, l_{2}$ and $r_{2}$ for notational convenience. Notice that the 
distribution of the limiting random variable defined by (3.9) is mixed normal with $\mathcal{F}$-conditional variance $\int_{0}^{1} v^{2}\left(\sigma_{u}\right) d u$. In the following we denote such a distribution by $M N\left(0, \int_{0}^{1} v^{2}\left(\sigma_{u}\right) d u\right)$.

Remark 3 Some of the assumptions can be relaxed, if all powers are even numbers. In particular, apart from the moment condition of $U$ we only have to postulate condition $(\mathrm{V})$. This is due to the fact that even moments of the pre-averaged noise process can be computed explicitly. One can see easily that these moments converge to the corresponding ones of a standard normal distribution fast enough.

Remark 4 A nice way to quantify the quality of estimators like $\hat{C}^{n}$ in contrast to their modulated bipower analogues is to have a look at its performance in a special setting. Suppose that the latent process is given by

$$
X_{t}=\sigma W_{t}
$$

for some positive constant $\sigma>0$. It is well-known from Gloter and Jacod [11] that one has an efficient parametric bound for the asymptotic variance of any estimator for $\sigma^{2}$, namely $8 \sigma^{3} \omega$. It was shown in Jacod et al. [14] that in this special case one can compute the conditional variance in Theorem 3 explicitly and obtains for the (probably most natural) weight function

$$
g(x)=(x \wedge(1-x))^{+}
$$

an optimal bound, which is roughly $8.5 \sigma^{3} \omega$ (by minimizing the conditional variance of $\hat{C}^{n}$ in $\theta$ ). This is not only rather close to the optimal bound, but also a huge improvement, since the related estimator discussed in Podolskij and Vetter [18] has an optimal variance of about $20 \sigma^{3} \omega$.

As mentioned earlier, we are able to estimate each entry $\int_{0}^{1} w_{i j}\left(\sigma_{u}\right) d u$ of the conditional covariance matrix. To this end, we fix $i$ and $j$ and choose some real number $\varpi \in\left(0, \frac{1}{4}\right)$. Moreover, we define

$$
\tilde{Z}_{m, i}^{n}=n^{\frac{l_{i}+r_{i}}{4}-\frac{1}{2}}\left|\bar{Z}_{m}^{n}\right|^{l_{i}}\left|\bar{Z}_{m+k_{n}}^{n}\right|^{r_{i}} 1_{\left\{\left|\bar{Z}_{m}^{n}\right|<n^{-\varpi},\left|\bar{Z}_{m+k_{n}}^{n}\right|<n^{-\varpi}\right\}}
$$

as well as

$$
\hat{\chi}_{m, l}^{n}=\frac{1}{2}\left(\tilde{Z}_{m, i}^{n}\left(\tilde{Z}_{m+l, j}^{n}-\tilde{Z}_{m+2 k_{n}, j}^{n}\right)+\tilde{Z}_{m, j}^{n}\left(\tilde{Z}_{m+l, i}^{n}-\tilde{Z}_{m+2 k_{n}, i}^{n}\right)\right),
$$


for any $0 \leq m \leq n-4 k_{n}+1$ and $1 \leq l<2 k_{n}$.

Note that the truncation in the definition of $\tilde{Z}_{m, i}^{n}$ is necessary in order to obtain an estimator for the variance in the central limit theorem, which is robust in the presence of jumps. It could be removed, if one wants to establish a feasible result only in model (1.1).

Lemma 1 If all conditions from Theorem 3 hold true and if $U$ further satisfies

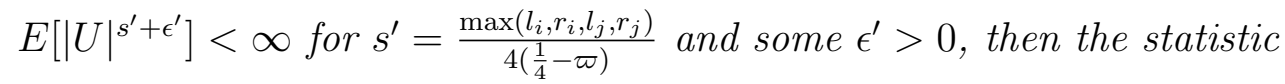

$$
\hat{w}_{i j}^{n}=\frac{2}{n^{\frac{1}{2}}} \sum_{m=0}^{n-4 k_{n}+1} \sum_{l=0}^{2 k_{n}-1} \hat{\chi}_{m, l}^{n}
$$

converges in probability to $\int_{0}^{1} w_{i j}\left(\sigma_{u}\right) d u$, both in model (1.1) and in model (1.3), as long as condition $(H)$ is satisfied.

Remark 5 The moment condition on $U$ in Lemma 1 is necessary to ensure that the probability of $n^{\frac{1}{4}}\left|\bar{U}_{i}^{n}\right|$ exceeding some threshold of the form $n^{\frac{1}{4}-\varpi}$ becomes sufficiently small. An alternative approach could involve less moments, but the additional assumption (A') in order to perform a similar type of Edgeworth expansion as in Theorem 3 .

We conclude this section with a second proposition on the asymptotic behaviour of bipower-type statistics in the general framework of (1.3). As in the case of stochastic convergence we will only show that the proposition from Theorem 3 holds under the presence of jumps as well, provided that the powers $l$ and $r$ are small enough. We will prove this result in the one-dimensional case only, since the extension to the bivariate setting is straightforward.

Before we can proceed with the statement of the result we need an additional condition on the semimartingale $X$, which is well-known from Jacod [12] as well.

(L- $q)$ : We have $(\mathrm{H})$ and the process $\delta(s, x)$ is predictable and left continuous with right limits. Moreover, there exists a family of functions $\gamma_{k}(x)$ and a sequence of stopping times $T_{k}$ converging to infinity almost surely such that

$$
|\delta(s, x)| \leq \gamma_{k}(x) \text { for all } s \leq T_{k}
$$


and

$$
\int_{\mathbb{R}} \Phi_{q}\left(\gamma_{k}(x)\right) d x<\infty
$$

with $q \in[0,2]$, any $k$, hold.

Note that (L- $q$ ) implies (L- $r$ ), whenever $q \leq r \leq 2$. The following claim is closely related to Theorem 6.2 in Jacod [13] in the no-noise case.

Theorem 4 Let $X$ be given by (1.3) and assume that (L-q) as well as (V), (V') and (A) are satisfied. If further $\frac{q}{2-q}<l_{1}, r_{1}<1$ and $E\left[|U|^{s+\epsilon}\right]<\infty$ for some $s \geq\left(3 \wedge 2\left(r_{1}+l_{1}\right)\right)$ and some $\epsilon>0$, then the stable convergence from Theorem 3 holds in the univariate setting. Precisely, we have

$$
n^{\frac{1}{4}}\left(B T\left(l_{1}, r_{1}\right)^{n}-B T\left(l_{1}, r_{1}\right)\right) \stackrel{\mathcal{D}_{s t}}{\longrightarrow} V\left(l_{1}, r_{1}\right),
$$

where $V\left(l_{1}, r_{1}\right)$ is the first component of the limiting variable $V\left(l_{1}, r_{1}, 0,0\right)$ as defined in Theorem 3.

\section{Testing for Jumps}

In order to derive a test for jumps we have to specify the hypotheses first. We assume throughout this paragraph that the underlying process $X$ is given by (3.4) for some choice of $a, \sigma$ and $\delta$, where $\delta \equiv 0$ corresponds to the setting in (1.1). Note however, that even if $\delta$ does not vanish and thus the process $X$ allows in principle for jumps, the realised path $s \mapsto X_{s}\left(\omega^{(0)}\right)$ does not have to have jumps at all. Obviously, in this case there is no way to tell whether the process comes from model (1.1) or from the more comprehensive model (1.3), since we are just able to distinguish between continuous and discontinuous paths of $X$. We therefore partition the set $\Omega$ into the following two subsets

$$
\Omega_{c}=\Omega_{c}^{(0)} \times \Omega^{(1)} \quad \text { and } \quad \Omega_{d}=\Omega_{d}^{(0)} \times \Omega^{(1)}
$$

with

$$
\begin{aligned}
& \Omega_{c}^{(0)}=\left\{\omega^{(0)}: s \mapsto X_{s}\left(\omega^{(0)}\right) \text { is continuous on }[0,1]\right\} \\
& \Omega_{d}^{(0)}=\left\{\omega^{(0)}: s \mapsto X_{s}\left(\omega^{(0)}\right) \text { is discontinuous on }[0,1]\right\}
\end{aligned}
$$


Fortunately, the properties of bipower-type statistics based on Itō diffusions and on Itō semimartingales without jumps are comparable. Thus $B T V^{n}$ from (3.6) can still be regarded as the right quantity to construct test for the presence of jumps from. If we choose $l_{1}=2, r_{1}=0$ and $l_{2}=r_{2}=1$, we obtain the representation

$$
B T V^{n}=\xi_{n}^{1}-\mu_{1}^{-2} \xi_{n}^{2}
$$

On the set $\Omega_{c}$ (under the null hypothesis of no jumps), exploiting the properties of stable convergence and the results of Theorem 3, this statistic converges stably in law, i.e

$$
n^{\frac{1}{4}} B T V^{n} \stackrel{\mathcal{D}_{s t}}{\longrightarrow} M N\left(0, \tau^{2}\right), \quad \tau^{2}=\int_{0}^{1} d^{2}\left(\sigma_{u}\right) d u,
$$

where $d^{2}$ is given by

$$
d^{2}=\left(\begin{array}{ll}
1 & -\mu_{1}^{-2}
\end{array}\right)\left(\begin{array}{ll}
w_{11} & w_{12} \\
w_{12} & w_{22}
\end{array}\right)\left(\begin{array}{c}
1 \\
-\mu_{1}^{-2}
\end{array}\right)=w_{11}-2 \mu_{1}^{-2} w_{12}+\mu_{1}^{-4} w_{22}
$$

We are now in a position to derive a test for jumps in the underlying process $X$, since we know from Lemma 1 how to estimate the conditional variance of the limiting process $V$ in Theorem 3. For each $\int_{0}^{1} w_{p q}\left(\sigma_{u}\right) d u$ we have a natural estimator using $\hat{w}_{p q}$, thus a consistent estimator

$$
\hat{\tau}_{n}^{2}=\hat{w}_{11}-2 \mu_{1}^{-2} \hat{w}_{12}+\mu_{1}^{-4} \hat{w}_{22}
$$

for $\tau^{2}$ can be defined as well. From the properties of stable convergence it follows that

$$
S^{n}=n^{\frac{1}{4}} \frac{B T V^{n}}{\hat{\tau}_{n}} \stackrel{\mathcal{D}_{s t}}{\longrightarrow} S,
$$

where $S$ follows a standard normal distribution and is independent of $\mathcal{F}$.

Under the alternative however, $B T V^{n}$ converges to a strictly positive quantity. Since moreover $\hat{\tau}_{n}^{2}$ was shown to be a robust estimator for $\tau^{2}$ even in the presence of noise, we see easily that $S^{n}$ tends to infinity, if the realisation of $X$ has a discontinuous path. Therefore, if we denote with $u_{\alpha}$ the $\alpha$-quantile of a standard normal distribution, we can define

$$
L^{n}(\alpha)=\left\{S^{n}>u_{1-\alpha}\right\}
$$

and obtain for the null hypothesis $H_{0}: \omega \in \Omega_{c}^{(0)}$ the following theorem: 
Theorem 5 Assume that the conditions from Theorem 3 and Lemma 1 hold true. Then the test defined by

$$
\varphi_{1}(\omega)= \begin{cases}1, & \omega \in L^{n}(\alpha) \\ 0, & \omega \notin L^{n}(\alpha)\end{cases}
$$

fulfills

$$
\lim _{n \rightarrow \infty} P\left(\varphi_{1}(\omega)=1 \mid \Omega_{c}\right)=\alpha
$$

in model (1.1), for any choice of the functions $a, \sigma$ and $\delta$, and has therefore the asymptotic level $\alpha$. Moreover, it is consistent, since

$$
\lim _{n \rightarrow \infty} P\left(\varphi_{1}(\omega)=1 \mid \Omega_{d}\right)=1
$$

holds in model (1.3) and under (H) as a result of Theorem 2 and Lemma 1, again for any choice of $a, \sigma$ and $\delta$ with $P\left(\Omega_{d}\right)>0$.

This result can be proven in the same way as Theorem 6 in Ait-Sahalia and Jacod [1], where it was shown that the asymptotic behaviour of Itō diffusions and of Itō semimartingales without jumps is essentially the same.

A second test can be based on the ratio of the two bipower-type statistics

$$
\frac{1}{\theta \psi_{2}} B T(2,0)^{n} \quad \text { and } \quad \frac{\mu_{1}^{-2}}{\theta \psi_{2}} B T(1,1)^{n} .
$$

Since under the null hypothesis both statistics converge to the same quantity, we have that

$$
B T R^{n}=\frac{B T(2,0)^{n}}{\mu_{1}^{-2} B T(1,1)^{n}} \stackrel{P}{\longrightarrow} 1 .
$$

Again with the aid of the generalised delta method, we conclude that

$$
n^{\frac{1}{4}}\left(B T R^{n}-1\right) \stackrel{\mathcal{D}_{s t}}{\longrightarrow} M N\left(0, v^{2}\right)
$$

with

$$
v^{2}=\frac{1}{B T(1,1)^{2}} \int_{0}^{1}\left(\mu_{1}^{4} w_{11}-2 \mu_{1}^{2} w_{12}+w_{22}\right)\left(\sigma_{u}\right) d u .
$$

By the same arguments as above a consistent estimator $\hat{v}_{n}^{2}$ for $v^{2}$ is given by

$$
\hat{v}_{n}^{2}=\frac{1}{\left(B T(1,1)^{n}\right)^{2}}\left(\mu_{1}^{4} \hat{w}_{11}-2 \mu_{1}^{2} \hat{w}_{12}+\hat{w}_{22}\right) .
$$


Therefore

$$
T^{n}=n^{\frac{1}{4}} \frac{\left(B T R^{n}-1\right)}{\hat{v}_{n}} \stackrel{\mathcal{D}_{s t}}{\longrightarrow} T
$$

for a standard normal $T$, which is independent of $\mathcal{F}$, and the following theorem can easily be derived.

Theorem 6 Let

$$
J^{n}(\alpha)=\left\{T^{n}>u_{1-\alpha}\right\} .
$$

Under the assumptions from Theorem 5 the test defined by

$$
\varphi_{2}(\omega)= \begin{cases}1, & \omega \in J^{n}(\alpha) \\ 0, & \omega \notin J^{n}(\alpha)\end{cases}
$$

has the asymptotic level $\alpha$ and is consistent as well.

\section{Appendix}

In the following we assume without loss of generality that $a$ and $\sigma$ as well as $a^{\prime}, \sigma^{\prime}, v^{\prime}$ and $F_{t}\left(\Psi_{2}\right)$ are bounded, which can be justified by a standard localisation procedure as explained in Barndorff-Nielsen et al. [4] and Jacod [12]. By the same arguments we can also replace the functions $\gamma_{k}$ in condition (L- $q$ ) by a bounded function $\gamma$. Constants appearing in the proofs are usually denoted by $C$ and may be dependent on the bounds of the various processes in $(1.1),(1.3)$ and (3.7). We write $C_{p}$, if these constants depend on an additional parameter $p$.

Some parts of the proofs will base upon the concepts and calculations presented in Podolskij and Vetter [18], hence we will refer to details illustrated therein quite often. Nevertheless, the proof of Theorem 3 is much more involved, due to the strong correlation between the summands in (2.8).

We show first that replacing $\psi_{1}^{n}$ and $\psi_{2}^{n}$ defined in (2.5) by its limits $\psi_{1}$ and $\psi_{2}$ does not affect both the consistency statement and the central limit theorem.

Lemma 2 It holds

$$
\int_{0}^{1}\left(\theta \psi_{2}^{n} \sigma_{u}^{2}+\frac{1}{\theta} \psi_{1}^{n} \omega^{2}\right)^{q} d u-\int_{0}^{1}\left(\theta \psi_{2} \sigma_{u}^{2}+\frac{1}{\theta} \psi_{1} \omega^{2}\right)^{q} d u=o_{p}\left(n^{-\frac{1}{4}}\right)
$$

for all $r, l \geq 0$ and all $q \geq 0$. 
Proof of Lemma 2 Using the mean value theorem and the boundedness of $\sigma$ one obtains the result, if both

$$
\psi_{1}-\psi_{1}^{n}=o\left(n^{-\frac{1}{4}}\right) \quad \text { and } \quad \psi_{2}-\psi_{2}^{n}=o\left(n^{-\frac{1}{4}}\right)
$$

can be shown. The first proposition follows from

$$
\begin{aligned}
\psi_{1}^{n} & =k_{n} \sum_{i=0}^{k_{n}-1}\left(g_{i+1}^{n}-g_{i}^{n}\right)^{2}=\frac{1}{k_{n}} \sum_{i=0}^{k_{n}-1}\left(g^{\prime}\left(\xi_{i}\right)\right)^{2} \text { for some } \xi_{i} \in\left[\frac{i}{k_{n}}, \frac{i+1}{k_{n}}\right] \\
& =\int_{0}^{1}\left(g^{\prime}(x)\right)^{2} d x+O\left(\frac{1}{k_{n}}\right)=\psi_{1}+o\left(n^{-\frac{1}{4}}\right),
\end{aligned}
$$

using (2.4) and the approximation error of a Riemann sum, since $g^{\prime}$ was assumed to be piecewise Lipschitz. The second assertion can be proven analogously.

\section{Proof of Theorem 1}

Prior to proving the stochastic convergence of the statistic $B T(l, r)^{n}$, note that it can be represented in the following way:

$$
B T(l, r)^{n}=n^{-\frac{1}{2}} \sum_{m=0}^{k_{n}-1} M B V(l, r)_{m}^{n},
$$

where $M B V(l, r)_{m}^{n}$ is given by

$$
M B V(l, r)_{m}^{n}=n^{\frac{l+r}{4}-\frac{1}{2}} \sum_{i=0}^{\left\lfloor\frac{n}{k_{n}}\right\rfloor-1}\left|\bar{Z}_{i k_{n}+m}^{n}\right|^{l}\left|\bar{Z}_{(i+1) k_{n}+m}^{n}\right|^{r} 1_{\left\{(i+1) k_{n}+m \leq n\right\}} .
$$

Each of these new statistics turns out to be a slight generalisation of the modulated bipower estimators as proposed in Podolskij and Vetter [18]. Therefore, Theorem 1 follows from the following proposition, which proves consistency of all quantites $M B V(l, r)_{m}^{n}$ in a uniform way.

Lemma 3 There exists a sequence of random variables $\gamma_{n}$ converging to zero in probability, for which

$$
M B V(l, r)_{m}^{n}-\frac{1}{\theta} B T(l, r) \leq \gamma_{n}
$$

holds for all $m \leq k_{n}$. 
Proof of Lemma 3 This proposition can be reduced to Lemma 2 and the corresponding assertion in Podolskij and Vetter [18], up to some minor changes. The crucial step in the proof of Theorem 1 therein is to assure that $n^{\frac{1}{4}} \bar{U}_{i}^{n}$ converges weakly to a normal distribution; however, this follows in our context, since Lindeberg's condition is satisfied due to the assumptions on $g$. Uniform convergence can be obtained, since the convergence to zero of any statistic $M B V(l, r)_{m}^{n}$ is obtained by the fact that $\sigma$ is supposed to be bounded and càdlàg, regardless of $m$.

\section{Proof of Theorem 2}

The first part of this theorem is shown in Theorem 3.2 in Jacod et al. [15]. For the second proposition observe that up to the choice of $\kappa$ the semimartingale $X$ can be written as follows:

$$
X_{t}=X_{0}+Q_{t}+N(\epsilon)_{t}+M(\epsilon)_{t}+B(\epsilon)_{t}
$$

for any $\epsilon \in(0, s], s$ small enough. The auxiliary processes are defined as

$$
\begin{aligned}
& N(\epsilon)_{t}=\left(x 1_{\{|x|>\epsilon\}}\right) \star \mu_{t}, \quad M(\epsilon)_{t}=\left(x 1_{\{|x| \leq \epsilon\}}\right) \star\left(\mu_{t}-\nu_{t}\right), \\
& B(\epsilon)_{t}=B_{t}-\left(\kappa(x) 1_{\{|x|>\epsilon\}}\right) \star \nu_{t}, \quad Q_{t}=\int_{0}^{t} \sigma_{s} d W_{s} .
\end{aligned}
$$

We set further

$$
Z_{t}^{\prime}=X_{0}+Q_{t}+U_{t} \quad \text { and } \quad Z_{t}^{\prime \prime}=N(\epsilon)_{t}+M(\epsilon)_{t}+B(\epsilon)_{t}
$$

We already know from Theorem 1 that $B T\left(Z^{\prime}, r, l\right)^{n}$ converges in probability to $B T(r, l)$, which forces us to prove

$$
E\left[\left|B T(Z, r, l)^{n}-B T\left(Z^{\prime}, r, l\right)^{n}\right|\right] \rightarrow 0
$$

We have

$$
B T(Z, r, l)^{n}-B T\left(Z^{\prime}, r, l\right)^{n}=\sum_{i=0}^{n-2 k_{n}+1} n^{\frac{l+r}{4}-1} \rho_{i}^{n}
$$

with

$$
\rho_{i}^{n}=\left|\bar{Z}_{i}^{n}\right|^{l}\left(\left|\bar{Z}_{i+k_{n}}^{n}\right|^{r}-\left|{\overline{Z^{\prime}}}_{i+k_{n}}^{n}\right|^{r}\right)+\left|{\overline{Z^{\prime}}}_{i+k_{n}}^{n}\right|^{r}\left(\left|\bar{Z}_{i}^{n}\right|^{l}-\left|{\overline{Z^{\prime}}}_{i}^{n}\right|^{l}\right)
$$

Recall (2.7). Since

$$
E\left[\left|\bar{Z}_{i}^{\prime}{ }^{n}\right|^{q} \mid \mathcal{F}_{\frac{i}{n}}\right] \leq C_{q} n^{-\frac{q}{4}}
$$


for any positive $q$ such that $E\left[|U|^{q}\right]<\infty$ and

$$
E\left[\left|{\overline{Z^{\prime \prime}}}_{i}^{n}\right|^{q} \mid \mathcal{F}_{\frac{i}{n}}\right] \leq C_{q} n^{-\frac{q}{4}}
$$

for all $0<q \leq 2$ and by taking successive conditional expectations, it suffices to show that

$$
\sup _{i} n^{\frac{q}{4}} E\left[\left.|| \bar{Z}_{i}^{n}\right|^{q}-\left|{\overline{Z^{\prime}}}_{i}^{n}\right|^{q}|| \mathcal{F}_{\frac{i}{n}}\right] \leq \alpha_{n}
$$

for some deterministic sequence $\alpha_{n}$ converging to zero and all $0<q<2$. However, it has been shown in the proof of equation (15) in Ait-Sahalia and Jacod [1] that this property follows from

$$
E\left[\left|\bar{Z}_{i}^{\prime \prime}\right|^{2} \wedge n^{-\frac{1}{2}} \mid \mathcal{F}_{\frac{i}{n}}\right] \leq n^{-\frac{1}{2}} \beta_{n}
$$

for another deterministic sequence $\beta_{n}$, which goes to zero. In order to prove (5.4) we define

$$
\alpha_{i}^{n}(y)=E\left[\int_{\frac{i}{n}}^{\frac{i+k_{n}}{n}} \int_{\{|x| \leq y\}} \Phi_{2}(x) F_{t}(d x) d t\right],
$$

which is bounded due to the condition on $F_{t}\left(\Phi_{2}\right)$ stated in $(\mathrm{H})$. Let us now study the impact of the last three summands in (5.3). Again by (2.7) we have

$$
\overline{M(\epsilon)}_{i}^{n} \leq C \int_{\frac{i}{n}}^{\frac{i+k_{n}}{n}} d M(\epsilon)_{s}=C \overline{\Delta_{i}^{n} M(\epsilon)},
$$

and a similar result holds for $N(\epsilon)$ and $B(\epsilon)$. Therefore, these quanitites can be treated as increments of processes over small intervals, whose properties have already been studied in the proof of Lemma 4.1 in Jacod [12]. We conclude from the observations therein that the following two inequalities are valid

$$
E\left[\left|\overline{M(\epsilon)}_{i}^{n}\right|^{2}\right] \leq C \alpha_{i}^{n}(\epsilon) \quad \text { and } \quad\left|\overline{B(\epsilon)}_{i}^{n}\right| \leq C \frac{k_{n}}{n \epsilon} .
$$

Moreover, we can prove

$$
P\left(\overline{\Delta_{i}^{n} N(\epsilon)} \neq 0\right) \leq C \epsilon^{-2} n^{-\frac{1}{2}}
$$

analogously to the related statement in Jacod [12] as well. Therefore, we can conclude along the lines of Lemma 5.12 in the same paper that

$$
E\left[\left|{\overline{Z^{\prime \prime}}}_{i}^{n}\right|^{2} \wedge \eta^{2} \mid \mathcal{F}_{\frac{i}{n}}\right] \leq C n^{-\frac{1}{2}}\left(\frac{\eta^{2}+n^{-\frac{1}{2}}}{\delta^{2}}+\Gamma(\delta)\right)
$$


holds for all $\eta>0$ and $\delta \in(0,1)$, and with $\Gamma(\delta) \rightarrow 0$ as $\delta \rightarrow 0$. This finishes the proof of (5.4), and Theorem 2 follows.

Before we come to the proof of Theorem 3, we introduce an auxiliary result on Edgeworth-type expansions for triangular arrays of random variables $X_{n, i}$, where the $X_{n, i}$ are independent, but not identically distributed. Recall first that the $\nu$-th cumulant $\kappa_{\nu}$ of a random variable $X$ is defined to be the coefficient of $\frac{1}{\nu !}(i t)^{\nu}$ in a power series expansion of the cumulant generating function $\log (\chi(t))$, that is

$$
\log (\chi(t))=\sum_{l=0}^{\infty} \frac{1}{l !} \kappa_{l}(i t)^{l}
$$

provided such a series exists, at least up to order $\nu$. In the case of a triangular array, each $X_{n, i}$ has different cumulants $\kappa_{\nu, n, i}$, which makes standard results on Edgeworth expansions unavailable. Nevertheless, we will state a result closely related to a theorem in Lahiri [17], for which we need some additional notation.

Consider a series of real constants $\left(\gamma_{i}\right)$. We then define for any integer $s$ the formal polynomial

$$
\tilde{P}_{s}\left(z:\left(\gamma_{i}\right)\right)=\sum_{m=1}^{s} \frac{1}{m !}\left(\sum_{j_{1}, \ldots, j_{m}}^{*} \prod_{i=1}^{m} \gamma_{j_{i}+2}\right) z^{2 m+s},
$$

where $\sum_{j_{1}, \ldots, j_{m}}^{*}$ denotes the sum over all $m$-tupels of positive integers $j_{1}, \ldots, j_{m}$ with

$$
\sum_{i=1}^{m} j_{i}=s
$$

and an empty sum is defined to be 1 . We see easily that the coefficients only involve such $\gamma_{i}$ with $i \leq s+2$. Moreover, $\tilde{P}_{s}$ is even, if and only if $s$ is even. We set further

$$
P_{s}\left(-\varphi:\left(\gamma_{i}\right)\right)=\tilde{P}_{s}\left(-D:\left(\gamma_{i}\right)\right) \varphi
$$

where $D$ is the differential operator, applied to the normal density $\varphi$. At last, we define $P_{s}\left(-\Phi:\left(\gamma_{i}\right)\right)$ to be the signed measure on $\mathbb{R}$, whose density is given by $P_{s}\left(-\varphi:\left(\gamma_{i}\right)\right)$. As usual, $P^{X}$ denotes the distribution of a random variable $X$.

By definition, $P_{0}\left(-\Phi:\left(\gamma_{i}\right)\right)$ is $\Phi$ itself, whereas any other measure $P_{s}\left(-\Phi:\left(\gamma_{i}\right)\right)$ has an even density for even $s$ and an odd density for odd $s$. The following Lemma 
is a refinement of Theorem 6.1 in Lahiri [17], which can be proven in the same way as Theorem 6.2 therein.

Lemma 4 Let $\left(X_{n, j}\right)$ be a triangular array of row-wise independent real-valued random variables $X_{n, 1}, \ldots, X_{n, n}$ with zero mean and $\frac{1}{n} \sum_{j=1}^{n} E\left[X_{n, j}^{2}\right]=1$ for each $n$. Suppose further that the following conditions are satisfied for some integer $s \geq 3$ and some $\delta \in\left(0, \frac{1}{2}\right)$ :

(i) $\lim _{n \rightarrow \infty} n^{-1} \sum_{j=1}^{n} E\left[\left|X_{n, j}\right|^{s} 1_{\left\{\left|X_{n, j}\right|>n^{\frac{1}{2}-\delta}\right\}}\right]=0$.

(ii) $\lim \sup _{n \rightarrow \infty} \bar{\rho}_{n, s}<\infty$ with $\bar{\rho}_{n, s}=\frac{1}{n} \sum_{j=1}^{n} E\left[\left|X_{n, j}\right|^{s}\right]$.

(iii) For some positive sequence $\left(\eta_{n}\right)$ with $\eta_{n}=o\left(n^{-\frac{s-2}{2}}\right)$ we have

$$
\limsup _{n \rightarrow \infty} \sup \left\{\left|\chi_{n}^{j}(t)\right| ; 16\left(\bar{\rho}_{n, 3}\right)^{-1} \leq|t| \leq \eta_{n}^{-4}, j=1, \ldots, n\right\}<1
$$

where $\chi_{n}^{j}$ denotes the characteristic function of $X_{n, j}$.

Then for every real-valued, Borel-measurable function $f$ satisfying

$$
M_{s}(f)=\sup _{x \in \mathbb{R}}\left(1+|x|^{2\left\lfloor\frac{s}{2}\right\rfloor}\right)^{-1}|f(x)|<\infty
$$

we have

$$
\left|\int f d\left(P^{S_{n}}-\sum_{r=0}^{s-2} n^{-\frac{r}{2}} P_{r}\left(-\Phi:\left(\bar{\kappa}_{\nu, n}\right)\right)\right)\right| \leq C M_{s}(f) \delta_{n}+C_{s} \bar{\omega}\left(2 \eta_{n} ; f, \Phi\right),
$$

where $S_{n}=n^{-\frac{1}{2}} \sum_{j=1}^{n} X_{n, j}, \bar{\kappa}_{\nu, n}$ is the average $\nu$-th cumulant of $X_{n, j}$ for $j=1, \ldots, n$, $C$ and $C_{s}$ are suitable constants, $\delta_{n}=o\left(n^{-\frac{s-2}{2}}\right)$ and

$$
\bar{\omega}(\epsilon ; f, \Phi)=\int \omega_{f}(\epsilon, x) \varphi(x) d x, \quad \omega_{f}(\epsilon, x)=\sup _{y, z \in(x-\epsilon, x+\epsilon)}|f(y)-f(z)| .
$$

(5.5) holds uniformly over a class of triangular arrays, as long as the conditions (i) - (iii) hold uniformly as well.

Note that the existence of the $s$-th moment implies that all cumulants up to order $s$ exist as well. Therefore, any $P_{r}\left(-\Phi:\left(\bar{\kappa}_{\nu, n}\right)\right)$ is well-defined for $r \leq s-2$.

Lemma 4 can be used to prove that the error due to the approximation of moments of pre-averaged statistics by the corresponding ones of a normal distribution 
is of a smaller order than $n^{-\frac{1}{4}}$. Let us first introduce some further notation. For any $m \leq i$ we define the class of random variables

$$
\bar{Y}_{i, m}^{n}=\sum_{j=1}^{k_{n}-1} g_{j}^{n}\left(\sigma_{\frac{m}{n}} \Delta_{i+j}^{n} W+\Delta_{i+j}^{n} U\right)
$$

These quantities are approximations for the random variable $\bar{Z}_{i}^{n}$, since we exchanged the increments of $X$ over small intervals by the associated increments of the underlying Brownian motion $W$ times $\sigma$ evaluated at some time point $\frac{m}{n}$. Moreover, we set

$$
\eta_{i, m}^{n}=n^{\frac{l+r}{4}}\left|\bar{Y}_{i, m}^{n}\right|^{l}\left|\bar{Y}_{i+k_{n}, m}^{n}\right|^{r}
$$

for arbitrary non-negative powers $l$ and $r$.

Lemma 5 Let $X$ be given by (1.1) and assume that $U$ satisfies condition (A) as well as $E\left[|U|^{s+\epsilon}\right]<\infty$ for some $s \geq(3 \wedge 2(r+l))$ and some $\epsilon>0$. Moreover, we have either ( $\left.V^{\prime}\right)$ or $\left(A^{\prime}\right)$. Then

$$
E\left[\eta_{i, m}^{n} \mid \mathcal{F}_{\frac{i}{n}}\right]=\mu_{r} \mu_{l}\left(\sigma_{\frac{m}{n}}^{2} \theta \psi_{2}+\frac{1}{\theta} \psi_{1} \omega^{2}\right)^{\frac{l+r}{2}}+o_{p}\left(n^{-\frac{1}{4}}\right),
$$

uniformly in $i$ and $m$.

Proof of Lemma 5 Note first that without loss of generality is suffices to prove the result in the case $r=0$, since $\bar{Y}_{i, m}^{n}$ and $\bar{Y}_{i+k_{n}, m}^{n}$ are conditionally independent. We set $f(x)=|x|^{l}$ and find that

$$
E\left[\eta_{i, m}^{n} \mid \mathcal{F}_{\frac{i}{n}}\right]=\int f d P^{U_{i, m, n}^{\prime}}
$$

where

$$
U_{i, m, n}^{\prime}=n^{\frac{1}{4}} \sum_{j=1}^{k_{n}}\left(\frac{1}{\sqrt{n}} \sigma \frac{m}{n} g_{j}^{n} N_{i+j}+\left(g_{j-1}^{n}-g_{j}^{n}\right) U_{i+j}\right)=: k_{n}{ }^{-\frac{1}{2}} \sum_{j=1}^{k_{n}} \Gamma_{i+j}^{m, n},
$$

$\sigma_{\frac{m}{n}}$ can be treated as a non-random quantity and the $N_{l}$ are i.i.d. standard normal variables. By definition, $U_{i, m, n}^{\prime}$ has mean zero and a variance of

$$
\tau_{m, n}^{2}=\sigma_{\frac{m}{n}}^{2} \frac{k_{n}}{n^{\frac{1}{2}}} \psi_{2}^{n}+\frac{n^{\frac{1}{2}}}{k_{n}} \psi_{1}^{n} \omega^{2},
$$


which converges to $\sigma_{\frac{m}{n}}^{2} \theta \psi_{2}+\frac{1}{\theta} \psi_{1} \omega^{2}$ for any fixed $m$. Therefore (5.7) follows from a similar argument as in the proof of Lemma 2, once we have proven that

$$
\left|\int f d\left(P^{\bar{U}_{i, m, n}}-\Phi\right)\right|=o\left(n^{-\frac{1}{4}}\right)
$$

uniformly in $i$ and $m$, where ${\overline{U^{\prime}}}_{i, m, n}=\frac{U_{i, m, n}^{\prime}}{\tau_{m, n}}$ is a standardised sum with mean zero and unit variance.

Let us first add some comments on Lemma 4. For the choice of $f$ as above, a simple calculation shows that

$$
\bar{\omega}(\epsilon ; f, \Phi)=O(\epsilon)
$$

We conclude that whenever Lemma 4 holds,

$$
\left|\int f d\left(P^{S_{n}}-\sum_{r=0}^{s-2} n^{-\frac{r}{2}} P_{r}\left(-\Phi:\left(\bar{\kappa}_{\nu, n}\right)\right)\right)\right|=o\left(n^{-\frac{s-2}{2}}\right)
$$

follows for such a function $f$, provided the conditions $s \geq 3$ and $\left\lfloor\frac{s}{2}\right\rfloor \geq \frac{l}{2}$ are satisfied.

For our purposes it is sufficient to use the expansion to first order. If we assume that the conditions for an application of Lemma 4 are satisfied for an integer $s$ as specified in Lemma 5, we can conclude

$$
\left|\int f d\left(P^{\overline{U^{\prime}} i, m, n}-\Phi-k_{n}{ }^{-\frac{1}{2}} P_{1}\left(-\Phi:\left(\bar{\kappa}_{1, i, m, n}\right)\right)\right)\right|=o\left(k_{n}{ }^{-\frac{1}{2}}\right)=o\left(n^{-\frac{1}{4}}\right),
$$

where $\bar{\kappa}_{\nu, i, m, n}$ denotes the average $\nu$-th cumulant of $\frac{\Gamma_{i+j}^{m, n}}{\tau_{m, n}}, j \leq k_{n}$. Since $P_{1}(-\Phi$ : $\left.\left(\bar{\kappa}_{1, i, m, n}\right)\right)$ has an odd density and $f$ is an even function, we have

$$
\int f d P_{1}\left(-\Phi:\left(\bar{\kappa}_{1, i, m, n}\right)\right)=0
$$

and (5.8) follows. We are therefore left to prove that the assumptions $(i)-($ iii $)$ on $U_{i, m, n}^{\prime}$ are fulfilled, uniformly in $i$ and $m$.

$(i)$ and $(i i)$ follow easily from an application of Hölder's inequality, whereas in order to prove assumption $(i i i)$ we fix $i$ and $m$ and denote by $\chi_{j}^{n}$ the characteristic function of $\Gamma_{i+j}^{m, n} / \tau_{m, n}$. With

$$
\Gamma_{i+j}^{\prime m, n}=n^{-\frac{1}{4}} k_{n}^{\frac{1}{2}} \sigma \frac{m}{n} g_{j}^{n} N_{i+j} \quad \text { and } \quad \Gamma_{i+j}^{\prime \prime n}=n^{\frac{1}{4}} k_{n}^{\frac{1}{2}}\left(g_{j-1}^{n}-g_{j}^{n}\right) U_{i+j}
$$


we have

$$
\begin{aligned}
\left|\chi_{j}^{n}(t)\right| & =\left|E\left[\exp \left(i t \Gamma_{i+j}^{m, n} / \tau_{m, n}\right)\right]\right| \\
& =\left|E\left[\exp \left(i t \Gamma_{i+j}^{\prime m, n} / \tau_{m, n}\right)\right]\right|\left|E\left[\exp \left(i t \Gamma_{i+j}^{\prime \prime n} / \tau_{m, n}\right)\right]\right|
\end{aligned}
$$

since $\Gamma_{i+j}^{\prime m, n}$ and $\Gamma_{i+j}^{\prime \prime n}$ are independent.

If we additionally have ( $\left.\mathrm{V}^{\prime}\right)$, we can assume that $\sigma$ is bounded away from zero as well. This is again justified by a standard localising procedure, since one can find a sequence of stopping times $T_{k}$, converging to infinity, such that $\sigma_{s}^{2}>C_{k}>0$ for all $s<T_{k}$. Thus we can use the fact that the latter term on the right hand side of (5.9) is bounded by one, whereas the first quantity is the absolute value of the characteristic function of a normal distribution with variance

$$
v_{m, j, n}^{2}=\frac{k_{n} \sigma_{\frac{m}{n}}^{2}\left(g_{j}^{n}\right)^{2}}{n^{\frac{1}{2}} \tau_{m, n}^{2}} .
$$

Therefore we have

$$
\left|\chi_{j}^{n}(t)\right| \leq\left|E\left[\exp \left(i t \Gamma_{i+j}^{\prime m, n} / \tau_{m, n}\right)\right]\right|=\exp \left(-\frac{v_{m, j, n}^{2} t^{2}}{2}\right) .
$$

Since $v_{m, j, n}^{2}$ is now bounded from below, (iii) follows immediately. On the other hand, if we impose assumption (A'), we can focus on the characteristic function of $\Gamma_{i+j}^{\prime \prime}$. We set $h_{j}^{n}=n^{\frac{1}{4}} k_{n}^{\frac{1}{2}}\left(g_{j-1}^{n}-g_{j}^{n}\right)$ and obtain

$$
\left|\chi_{j}^{n}(t)\right| \leq\left|E\left[\exp \left(i t \Gamma_{i+j}^{\prime \prime n} / \tau_{m, n}\right)\right]\right|=\left|E\left[\exp \left(i\left(h_{j}^{n} / \tau_{m, n}\right) t U_{i+j}\right)\right]\right| .
$$

Since $h_{j}^{n} / \tau_{m, n}$ is bounded both from above and below, uniformly in $m, j$ and $n$, we readily obtain the result.

\section{Proof of Theorem 3}

Here we will use the same "small blocks - big blocks"-technique as presented in Jacod et al. [14], which unfortunately needs a lot of additional notation. Precisely, we first choose an integer $p$, which later will go to infinity, and partition the $n$ observations into several subsets: Set

$$
a_{i}(p)=2 i(p+1) k_{n} \quad \text { and } \quad b_{i}(p)=2 i(p+1) k_{n}+2 p k_{n}
$$

and let $A_{i}(p)$ denote the set of integers $l$ satisfying $a_{i}(p) \leq l<b_{i}(p)$ and $B_{i}(p)$ the integers between the two sets $A_{i}(p)$ and $A_{i+1}(p)$, namely those fulfilling $b_{i}(p) \leq l<$ 
$a_{i+1}(p)$. We further define $j_{n}(p)$ to be the largest integer $j$ such that $b_{j}(p) \leq n$ holds (that means: $A_{i}(p)$ and $B_{i}(p)$ can be accomodated in the set $1, \ldots, n j_{n}(p)+1$ times each), which gives the identity

$$
j_{n}(p)=\left\lfloor\frac{n}{2 k_{n}(p+1)}\right\rfloor-1
$$

Moreover, we use the notation $i_{n}(p)=2\left(j_{n}(p)+1\right)(p+1) k_{n}$.

We set further

$$
\begin{aligned}
\Upsilon_{j, m}^{n} & =\left|\bar{Y}_{j, m}^{n}\right|^{l_{1}}\left|\bar{Y}_{j+k_{n}, m}^{n}\right|^{r_{1}}-E\left[\left|\bar{Y}_{j, m}^{n}\right|^{l_{1}}\left|\bar{Y}_{j+k_{n}, m}^{n}\right|^{r_{1}} \mid \mathcal{F}_{\frac{m}{n}}\right], \\
\Upsilon_{j, m}^{\prime n} & =\left|\bar{Y}_{j, m}^{n}\right|^{l_{2}}\left|\bar{Y}_{j+k_{n}, m}^{n}\right|^{r_{2}}-E\left[\left|\bar{Y}_{j, m}^{n}\right|^{l_{2}}\left|\bar{Y}_{j+k_{n}, m}^{n}\right|^{r_{2}} \mid \mathcal{F}_{\frac{m}{n}}\right]
\end{aligned}
$$

and define

$$
\tilde{Y}_{j}^{n}=\left\{\begin{array}{ll}
n^{\frac{l_{1}+r_{1}}{4}-\frac{1}{2}} \Upsilon_{j, a_{i}(p)}^{n}, & j \in A_{i}(p) \\
n^{\frac{l_{1}+r_{1}}{4}-\frac{1}{2}} \Upsilon_{j, b_{i}(p)}^{n}, & j \in B_{i}(p) \\
n^{\frac{l_{1}+r_{1}}{4}-\frac{1}{2}} \Upsilon_{j, i_{n}(p)}^{n}, & j \geq i_{n}(p)
\end{array} \quad \tilde{Y}_{j}^{\prime}{ }_{j}^{n}= \begin{cases}n^{\frac{l_{2}+r_{2}}{4}-\frac{1}{2} \Upsilon_{j, a_{i}(p)}^{\prime n},}, & j \in A_{i}(p) \\
n^{\frac{l_{2}+r_{2}}{4}-\frac{1}{2}} \Upsilon_{j, b_{i}(p)}^{\prime n}, & j \in B_{i}(p) \\
n^{\frac{l_{2}+r_{2}}{4}-\frac{1}{2} \Upsilon_{j, i_{n}(p)}^{\prime n},} & j \geq i_{n}(p)\end{cases}\right.
$$

as well as

$$
\begin{aligned}
\zeta(p, 1)_{j}^{n}=\sum_{l=a_{j}(p)}^{b_{j}(p)-1} \tilde{Y}_{l}^{n} & \text { and } & \zeta(p, 1)^{\prime n} & =\sum_{l=a_{j}(p)}^{b_{j}(p)-1} \tilde{Y}_{l}^{\prime}{ }_{l}^{n}, \\
\zeta(p, 2)_{j}^{n}=\sum_{l=b_{j}(p)}^{a_{j+1}(p)-1} \tilde{Y}_{l}^{\prime} & \text { and } & \zeta(p, 2)^{\prime n}{ }_{j}^{n} & =\sum_{l=b_{j}(p)}^{a_{j+1}(p)-1} \tilde{Y}_{l}^{\prime}{ }_{l}^{n} .
\end{aligned}
$$

We set at last

$$
\left.\begin{array}{lrl}
M(p)^{n}=n^{-\frac{1}{2}} \sum_{j=0}^{j_{n}(p)} \zeta(p, 1)_{j}^{n} & M(p)^{\prime n}=n^{-\frac{1}{2}} \sum_{j=0}^{j_{n}(p)} \zeta(p, 1)_{j}^{\prime n} \\
N(p)^{n}=n^{-\frac{1}{2}} \sum_{j=0}^{j_{n}(p)} \zeta(p, 2)_{j}^{n} & N(p)^{\prime n}=n^{-\frac{1}{2}} \sum_{j=0}^{j_{n}(p)} \zeta(p, 2)^{\prime n} \\
C(p)^{n}=n^{-\frac{1}{2}} \sum_{j=i_{n}(p)}^{n} \tilde{Y}_{j}^{n} & C(p)^{\prime n}=n^{-\frac{1}{2}} \sum_{j=i_{n}(p)}^{n} \tilde{Y}_{j}^{\prime n}
\end{array}\right\}
$$

and note that

$$
E\left[\zeta(p, 1)_{j}^{n} \mid \mathcal{F}_{\frac{a_{j}(p)}{n}}\right]=0=E\left[\zeta(p, 2)_{j}^{n} \mid \mathcal{F}_{\frac{b_{j}(p)}{n}}\right]
$$

by construction. The same property holds for the corresponding prime variables.

The outline of the proof is as follows: We will first show that

$$
n^{\frac{1}{4}}\left(B T\left(l_{1}, r_{1}\right)^{n}-B T\left(l_{1}, r_{1}\right)\right)=n^{\frac{1}{4}} H(p)^{n}+F(p)^{n}
$$


holds, where $F(p)^{n}$ has the property

$$
\lim _{p \rightarrow \infty} \limsup _{n \rightarrow \infty} P\left(\left|F(p)^{n}\right|>\epsilon\right)=0
$$

and $H(p)_{n}$ is given by

$$
H(p)^{n}=M(p)^{n}+R(p)^{n} \text { with } R(p)^{n}=N(p)^{n}+C(p)^{n} .
$$

In a second step we will prove

$$
\lim _{p \rightarrow \infty} \limsup _{n \rightarrow \infty} P\left(\left|n^{\frac{1}{4}} R(p)^{n}\right|>\epsilon\right)=0
$$

for each $\epsilon>0$. Similar results hold for $n^{\frac{1}{4}}\left(B T\left(l_{2}, r_{2}\right)^{n}-B T\left(l_{2}, r_{2}\right)\right)$ as well. These steps ensure that it is sufficient to derive a joint limit theorem for $M(p)^{n}$ and $M(p)^{\prime n}$ for any fixed $p$. The proof of this claim is given in the third step. Precisely, we will obtain

$$
n^{\frac{1}{4}}\left(M(p)^{n}, M(p)^{\prime n}\right) \stackrel{\mathcal{D}_{s t}}{\longrightarrow} V(p)=\int_{0}^{1} v\left(\sigma_{u}, p\right) d W_{u}^{\prime},
$$

where the $2 \times 2$-dimensional process $v\left(\sigma_{u}, p\right)$ is bounded and converges pointwise in $p$ to the limiting process $v\left(\sigma_{u}\right)$ as defined in Theorem 3. Therefore

$$
V(p) \stackrel{P}{\longrightarrow} V\left(l_{1}, r_{1}, l_{2}, r_{2}\right)=\int_{0}^{1} v\left(\sigma_{u}\right) d W_{u}^{\prime},
$$

which will finish the proof.

Lemma 6 It holds

$$
n^{\frac{1}{4}}\left(B T\left(l_{1}, r_{1}\right)^{n}-B T\left(l_{1}, r_{1}\right)\right)=n^{\frac{1}{4}} H(p)^{n}+F(p)^{n}
$$

where $F(p)^{n}$ satisfies (5.12).

Proof of Lemma 6 First, we introduce some auxiliary random variables. Let

$$
\xi_{i}^{n}=n^{\frac{l_{1}+r_{1}}{4}}\left|\bar{Z}_{i}^{n}\right|^{l_{1}}\left|\bar{Z}_{i+k_{n}}^{n}\right|^{r_{1}}
$$

and define

$$
\Lambda_{m}^{n}=\sum_{j=0}^{j_{n}(p)} \tilde{Y}_{a_{j}(p)+m}^{n}+\tilde{Y}_{i_{n}(p)+m}^{n} 1_{\left\{i_{n}(p)+m \leq n\right\}}
$$


as well as

$$
\Xi_{m}^{n}=n^{-\frac{1}{2}}\left(\sum_{j=0}^{j_{n}(p)} \xi_{a_{j}(p)+m}^{n}+\xi_{i_{n}(p)+m}^{n} 1_{\left\{i_{n}(p)+m \leq n\right\}}\right)
$$

for any $0 \leq m<2(p+1) k_{n}$.

We first rewrite the two statistics in the following way:

$$
\begin{aligned}
B T\left(l_{1}, r_{1}\right)^{n}-B T\left(l_{1}, r_{1}\right) & =n^{-\frac{1}{2}} \sum_{m=0}^{2(p+1) k_{n}-1}\left(\Xi_{m}^{n}-\frac{1}{2(p+1) \theta} B T\left(l_{1}, r_{1}\right)\right) \\
& +o_{p}\left(n^{-\frac{1}{4}}\right) \\
\text { and } H(p)^{n} & =n^{-\frac{1}{2}} \sum_{m=0}^{2(p+1) k_{n}-1} \Lambda_{m}^{n} .
\end{aligned}
$$

Thus we are left to prove

$$
\lim _{p \rightarrow \infty} \limsup _{n \rightarrow \infty} \sup _{m} P\left(\left|n^{\frac{1}{4}}\left(\left(2(p+1) \Xi_{m}^{n}-\frac{1}{\theta} B T\left(l_{1}, r_{1}\right)\right)-2(p+1) \Lambda_{m}^{n}\right)\right|>\epsilon\right)=0 .
$$

We see easily that the claim follows, once we have proven the following two equations:

$$
\begin{aligned}
& \lim _{p \rightarrow \infty} \limsup _{n \rightarrow \infty} \sup _{m} \\
& \quad P\left(\left|n^{\frac{1}{4}}\left(2(p+1) n^{-\frac{1}{2}} \sum_{j=0}^{j_{n}(p)} E\left[\eta_{a_{j}(p)+m, a_{j}(p)}^{n} \mid \mathcal{F}_{\frac{a_{j}(p)}{n}}\right]-\frac{1}{\theta} B T\left(l_{1}, r_{1}\right)\right)\right|>\epsilon\right)=0
\end{aligned}
$$

and

$$
\lim _{p \rightarrow \infty} \limsup _{n \rightarrow \infty} \sup _{m} P\left(2(p+1) n^{\frac{1}{4}}\left|\Xi_{m}^{n}-n^{-\frac{1}{2}} \sum_{j=0}^{j_{n}(p)} \eta_{a_{j}(p)+m, a_{j}(p)}^{n}\right|>\epsilon\right)=0 .
$$

The convergence in (5.16) can be concluded from an application of Lemma 5, (2.4) and the approximation error of a Riemann sum.

For the proof of (5.17), we will use related propositions in Barndorff-Nielsen et al. [4]. Note that by the same arguments as in their work the result follows from

$$
\lim _{p \rightarrow \infty} \limsup _{n \rightarrow \infty} \sup _{m} P\left(2(p+1) n^{-\frac{1}{4}} \sum_{j=0}^{j_{n}(p)} E\left[\mid \xi_{a_{j}(p)+m}^{n}-\eta_{a_{j}(p)+m}^{n} \| \mathcal{F}_{\frac{m k_{n}}{n}}\right]>\epsilon\right)=0 .
$$

A close look at the sections 7 and 8 of Barndorff-Nielsen et al. [4] shows that the proof of this claim works in the same way, provided one uses assumption (A) in two 
places. One has to define a quantity similar to the one in (7.11), whose absolute moments have to exist for all powers $s \in[0,1)$, which holds in our context due to (ii) in (A). Secondly, one needs the symmetry of $U$ to conclude similarly as in part (4) of Section 8.

We start our computations on $H(p)^{n}$ with a simple result on $C(p)^{n}$.

Lemma 7 We have

$$
\lim _{p \rightarrow \infty} \limsup _{n \rightarrow \infty} P\left(\left|C(p)^{n}\right|>\epsilon\right)=0 .
$$

Proof of Lemma 7 For any fixed $p \geq 1$ is the number of summands in $C(p)^{n}$ bounded above by $C_{p} n^{\frac{1}{2}}$. Moreover, each summand as well as the factor in front of the sum is of order $n^{-\frac{1}{2}}$. This gives the result.

The next auxiliary result gives information about the order of $N(p)^{n}$, this time depending on the integer $p$.

Lemma 8 Assume that $p$ is fixed. Then

$$
E\left[\left(n^{\frac{1}{4}} N(p)^{n}\right)^{2}\right] \leq \frac{C}{p}
$$

is valid.

Proof of Lemma 8 We know from (5.11) that the process

$$
L_{k}^{n}=n^{-\frac{1}{2}} \sum_{j=0}^{k} \zeta(p, 2)_{j}^{n}
$$

is a martingale with respect to the filtration $\mathcal{G}(p)_{j}^{n}=\mathcal{F}_{\frac{b_{j}(p)}{n}}$, which implies

$$
E\left[\left(n^{\frac{1}{4}} N(p)^{n}\right)^{2}\right] \leq 4 n^{-\frac{1}{2}} \sum_{j=0}^{j_{n}(p)} E\left[\left(\zeta(p, 2)_{j}^{n}\right)^{2}\right]
$$

via Doob's inequality. Due to the assumptions on $a$ and $\sigma$ we have

$$
E\left[\left(\tilde{Y}_{j}^{n}\right)^{2}\right] \leq C n^{-1}
$$


independent of $j$ and $p$. This yields

$$
E\left[\left(\zeta(p, 2)_{j}^{n}\right)^{2}\right] \leq C
$$

Hence we obtain the result, since $j_{n}(p) \leq C \frac{\sqrt{n}}{p}$ holds.

It remains to show the stable convergence of $n^{\frac{1}{4}}\left(M(p)^{n}, M(p)^{\prime n}\right)$.

Lemma 9 For any fixed $p \geq 2$ we have

$$
n^{\frac{1}{4}}\left(M(p)^{n}, M(p)^{\prime n}\right) \stackrel{\mathcal{D}_{s t}}{\longrightarrow} \int_{0}^{1} v\left(\sigma_{u}, p\right) d W_{u}^{\prime},
$$

where $W^{\prime}$ is a standard Brownian motion independent of $\mathcal{F}$. We have

$$
v^{t}\left(\sigma_{u}, p\right) v\left(\sigma_{u}, p\right)=\left(\begin{array}{ll}
w_{11} & w_{12} \\
w_{12} & w_{22}
\end{array}\right)\left(\sigma_{u}, p\right)
$$

with

$$
w_{i j}\left(\sigma_{u}, p\right)=\theta \int_{0}^{2}\left(2+\frac{1-s}{p}\right) h_{i j}\left(\sigma_{u}, t, f(s)\right) d s
$$

where $h_{i j}$ was defined in (3.8). Moreover, $v^{t}\left(\sigma_{u}, p\right) v\left(\sigma_{u}, p\right)$ converges pointwise in $p$ to $v^{t}\left(\sigma_{u}\right) v\left(\sigma_{u}\right)$.

Proof of Lemma 9 We define $\zeta(p)_{j}^{n}=\left(\zeta(p, 1)_{j}^{n}, \zeta(p, 1)_{j}^{\prime n}\right)$. Due to Theorem IX 7.28 in Jacod and Shiryaev [16] the following conditions have to be shown

$$
\begin{aligned}
& n^{-\frac{1}{2}} \sum_{j=0}^{j_{n}(p)} E\left[\left(\zeta(p)_{j}^{n}\right)^{t} \zeta(p)_{j}^{n} \mid \mathcal{F}_{\frac{a_{j}(p)}{n}}\right] \stackrel{P}{\longrightarrow} \int_{0}^{1} v^{t}\left(\sigma_{u}, p\right) v\left(\sigma_{u}, p\right) d u \\
& n^{-1} \sum_{j=0}^{j_{n}(p)} E\left[\| \zeta(p)_{j}^{n}||^{4} \mid \mathcal{F}_{\frac{a_{j}(p)}{n}}\right] \stackrel{P}{\longrightarrow} 0 \\
& n^{-\frac{1}{4}} \sum_{j=0}^{j_{n}(p)} E\left[\zeta(p)_{j}^{n} \Delta W(p)_{j}^{n} \mid \mathcal{F}_{\frac{a_{j}(p)}{n}}\right] \stackrel{P}{\longrightarrow} 0 \\
& n^{-\frac{1}{4}} \sum_{j=0}^{j_{n}(p)} E\left[\zeta(p)_{j}^{n} \Delta N(p)_{j}^{n} \mid \mathcal{F}_{\frac{a_{j}(p)}{n}}\right] \stackrel{P}{\longrightarrow} 0
\end{aligned}
$$


with $\Delta V(p)_{j}^{n}=V_{b_{j}(p)}^{n}-V_{a_{j}(p)}^{n}$ for any process $V$ and (5.25) holding for any bounded martingale $N$ being orthogonal to $W$.

(5.24) is obvious, since $\zeta(p)_{j}^{n}$ is an even functional in $W$ and the distribution of $U$ is symmetric. Moreover, with the same arguments as in the proof of Lemma 8 we obtain

$$
E\left[\left\|\zeta(p)_{j}^{n}\right\|^{4}\right] \leq C
$$

which implies (5.23). (5.25) can be shown by the same methods as in the proof of Lemma 5.7. in Jacod et al. [14].

We prove (5.22) only for the first entry of the matrix, since analogous proofs hold in the other cases. Note first that Lemma 5 secures that we may proceed as if $U$ were normally distributed. We apply the fact that $\tilde{Y}_{i}^{n}$ and $\tilde{Y}_{l}^{n}$ are conditionally independent for $|i-l| \geq 2 k_{n}$, from which

$$
\begin{aligned}
E\left[\left(\zeta(p, 1)_{j}^{n}\right)^{2} \mid \mathcal{F}_{\frac{a_{j}(p)}{n}}\right] & =2 \sum_{l=a_{j}(p)}^{b_{j}(p)-1} \sum_{i=l}^{b_{j}(p)-1} E\left[\tilde{Y}_{i}^{n} \tilde{Y}_{l}^{n} \mid \mathcal{F}_{\frac{a_{j}(p)}{n}}\right]+O_{p}\left(n^{-1}\right) \\
& =2 \sum_{l=a_{j}(p)}^{b_{j}(p)-2 k_{n}-1} \sum_{i=l}^{l+2 k_{n}-1} E\left[\tilde{Y}_{i}^{n} \tilde{Y}_{l}^{n} \mid \mathcal{F}_{\frac{a_{j}(p)}{n}}\right] \\
& +2 \sum_{l=b_{j}(p)-2 k_{n}}^{b_{j}(p)-1} \sum_{i=l}^{b_{j}(p)-1} E\left[\tilde{Y}_{i}^{n} \tilde{Y}_{l}^{n} \mid \mathcal{F}_{\frac{a_{j}(p)}{n}}\right]+O_{p}\left(n^{-1}\right) \\
& =: \quad \vartheta_{1}^{n}\left(\sigma_{\frac{a_{j}(p)}{n}}, p\right)+\vartheta_{1}^{\prime n}\left(\sigma_{\frac{a_{j}(p)}{n}}, p\right)+O_{p}\left(n^{-1}\right)
\end{aligned}
$$

follows. By construction, the conditional expectation of $\tilde{Y}_{i}^{n}$ and $\tilde{Y}_{l}^{n}$ depends only on $|i-l|$ and can be expressed in terms of $h_{11}$, which was introduced in (3.8). Thus we have for $i, l \in A_{j}(p)$

$$
E\left[\tilde{Y}_{i}^{n} \tilde{Y}_{l}^{n} \mid \mathcal{F}_{\frac{a_{j}(p)}{n}}\right]=E\left[\tilde{Y}_{a_{j}(p)}^{n} \tilde{Y}_{a_{j}(p)+|i-l|}^{n} \mid \mathcal{F}_{\frac{a_{j}(p)}{n}}\right]=\frac{1}{n} h_{11}\left(\sigma_{\frac{a_{j}(p)}{n}}, t_{n}, f^{n}\left(\frac{|i-l|}{k_{n}}\right)\right)
$$


with $t_{n}=\left(\frac{k_{n}}{n^{\frac{1}{2}}} \psi_{2}^{n}, \frac{n^{\frac{1}{2}}}{k_{n}} \psi_{1}^{n}\right)$ and

$$
\begin{aligned}
& f_{1}^{n}(s)=n^{-\frac{1}{2}} \sum_{j=0}^{k_{n}(1-s)} g_{j}^{n} g_{j+s k_{n}}^{n}, \\
& f_{2}^{n}(s)=n^{\frac{1}{2}} \sum_{j=0}^{k_{n}(1-s)}\left(g_{j}^{n}-g_{j+1}^{n}\right)\left(g_{j+s k_{n}}^{n}-g_{j+1+s k_{n}}^{n}\right) \\
& f_{3}^{n}(s)=n^{-\frac{1}{2}} \sum_{j=0}^{k_{n}(2-s)} g_{j}^{n} g_{j+s k_{n}-k_{n}}^{n} \\
& f_{4}^{n}(s)=n^{\frac{1}{2}} \sum_{j=0}^{k_{n}(2-s)}\left(g_{j}^{n}-g_{j+1}^{n}\right)\left(g_{j+s k_{n}-k_{n}}^{n}-g_{j+s k_{n}-k_{n}+1}^{n}\right) .
\end{aligned}
$$

We can conclude that

$$
n^{-\frac{1}{2}} \sum_{j=0}^{j_{n}(p)} \vartheta_{1}^{n}\left(\sigma_{\frac{a_{j}(p)}{n}}, p\right)=(4 p-2) \frac{k_{n}}{n^{\frac{3}{2}}} \sum_{j=0}^{j_{n}(p)} \sum_{i=0}^{2 k_{n}-1} h_{11}\left(\sigma_{\frac{a_{j}(p)}{n}}, t_{n}, f^{n}\left(\frac{i}{k_{n}}\right)\right)
$$

holds. We will show that the quantity on the right side converges in probability to

$$
\left(2-\frac{1}{p}\right) \theta \int_{0}^{1} \int_{0}^{2} h_{11}\left(\sigma_{u}, t, f(s)\right) d s d u=: \int_{0}^{1} \int_{0}^{2} \rho(u, s) d s d u .
$$

Remember that $t$ and $f$ were defined following Theorem 3. In order to prove this proposition we have to take a closer look at the function $h_{11}$. Note first that the random vector $H$ in the definition of $h_{11}$ follows a representation

$$
H=\Sigma(x, y, z) U
$$

where $U \sim \mathcal{N}_{4}(0, I)$ and $\Sigma(x, y, z)$ is a lower triangular matrix, which is continuous in all arguments. Since due to Lebesgue's theorem $\Sigma \mapsto E[\eta(\Sigma U)]$ is for all functions $\eta$, which are continuous and of at most polynomial growth, a continuous mapping as well, we readily obtain that $h_{11}$ itself is continuous. Therefore, and since $\sigma$ was assumed to be bounded, we deduce that

$$
\rho_{n}(u, s):=\left(2-\frac{1}{p}\right) \frac{j_{n}(p)}{m_{n}(p)} \frac{k_{n}}{n^{\frac{1}{2}}} h_{11}\left(\sigma_{\frac{\left\lfloor u m_{n}(p)\right\rfloor}{m_{n}(p)}}, t_{n}, f^{n}\left(\frac{\left\lfloor k_{n} s\right\rfloor}{k_{n}}\right)\right)
$$

with $m_{n}(p)=\frac{n}{2 k_{n} p}$ is itself bounded. Since

$$
(4 p-2) \frac{k_{n}}{n^{\frac{3}{2}}} \sum_{j=0}^{j_{n}(p)} \sum_{i=0}^{2 k_{n}-1} h_{11}\left(\sigma_{\frac{a_{j}(p)}{n}}, t_{n}, f^{n}\left(\frac{i}{k_{n}}\right)\right)=\int_{0}^{1} \int_{0}^{2} \rho_{n}(u, s) d s d u
$$


its convergence to the quantity defined in (5.26) follows from Lebesgue's theorem, as long as $\rho_{n}$ converges pointwise to $\rho$ for almost all $(u, s) \in[0,1] \times[0,2]$. However, this follows from both (2.4) and (5.10) and from the fact that $f^{n}$ is càdlàg and converges pointwise to $f, \sigma$ is càdlàg as well and $t_{n}$ converges to $t$.

A similar reasoning yields

$$
\begin{aligned}
n^{-\frac{1}{2}} \sum_{j=0}^{j_{n}(p)} \vartheta_{1}^{\prime n}\left(\sigma_{\frac{a_{j}(p)}{n}}, p\right) & =n^{-\frac{3}{2}} \sum_{j=0}^{j_{n}(p)} \sum_{i=0}^{2 k_{n}-1}\left(4 k_{n}-2 i\right) h_{11}\left(\sigma_{\frac{a_{j}(p)}{n}}, t_{n}, f^{n}\left(\frac{i}{k_{n}}\right)\right) \\
& \stackrel{P}{\longrightarrow} \frac{\theta}{p} \int_{0}^{1} \int_{0}^{2}(2-s) h_{11}\left(\sigma_{u}, t, f(s)\right) d s d u .
\end{aligned}
$$

Hence (5.22) follows with the first entry of $v^{t}\left(\sigma_{u}, p\right) v\left(\sigma_{u}, p\right)$ being equal to

$$
\theta \int_{0}^{2}\left(2+\frac{1-s}{p}\right) h_{11}\left(\sigma_{u}, t, f(s)\right) d s .
$$

The convergence stated in (5.15) can now be concluded easily. The processes $w_{12}$ and $w_{22}$ as the other entries of the matrix $v^{t} v$ are obtained by the same arguments.

Proof of Lemma 1 Without loss of generality we prove Lemma 1 for $i=j=1$. Recall the notation from the proof of Theorem 2 , such that we can write

$$
Z_{t}=Z_{t}^{\prime}+Z_{t}^{\prime \prime}
$$

where the first process basically consists of the Brownian part of the semimartingale plus the noise process and the second process contains the drift part and the jump part of the semimartingale. We define the random quantities

$$
\tilde{\chi}_{i, j}^{n}=n^{\frac{r_{1}+l_{1}}{2}-1}\left|\bar{Y}_{i, i}^{n}\right|^{l_{1}}\left|\bar{Y}_{i+k_{n}, i}^{n}\right|^{r_{1}}\left(\left|\bar{Y}_{i+j, i}^{n}\right|^{l_{1}}\left|\bar{Y}_{i+k_{n}+j, i}^{n}\right|^{r_{1}}-\left|\bar{Y}_{i+2 k_{n}, i}^{n}\right|^{l_{1}}\left|\bar{Y}_{i+3 k_{n}, i}^{n}\right|^{r_{1}}\right)
$$

and note from standard arguments that the stochastic convergence

$$
\frac{2}{n^{\frac{1}{2}}} \sum_{m=0}^{n-4 k_{n}+1} \sum_{l=0}^{2 k_{n}-1} E\left[\tilde{\chi}_{m, l}^{n} \mid \mathcal{F}_{\frac{i}{n}}\right] \stackrel{P}{\longrightarrow} \int_{0}^{1} w_{i j}\left(\sigma_{u}\right) d u
$$

holds. With

$$
\check{\chi}_{i, j}^{n}=n^{\frac{r_{1}+l_{1}}{2}-1}\left|{\overline{Z^{\prime}}}_{i}^{n}\right|^{l_{1}}\left|{\overline{Z^{\prime}}}_{i+k_{n}}^{n}\right|^{r_{1}}\left(\left|{\overline{Z^{\prime}}}_{i+j}^{n}\right|^{l_{1}}\left|{\overline{Z^{\prime}}}_{i+k_{n}+j}^{n}\right|^{r_{1}}-\left|{\overline{Z^{\prime}}}_{i+2 k_{n}}^{n}\right|^{l_{1}}\left|{\overline{Z^{\prime}}}_{i+3 k_{n}}^{n}\right|^{r_{1}}\right)
$$


it remains to prove that both

$$
\frac{2}{n^{\frac{1}{2}}} \sum_{m=0}^{n-4 k_{n}+1} \sum_{l=0}^{2 k_{n}-1} E\left[\check{\chi}_{m, l}^{n}-\tilde{\chi}_{m, l}^{n} \mid \mathcal{F}_{\frac{i}{n}}\right] \stackrel{P}{\longrightarrow} 0
$$

and

$$
\frac{2}{n^{\frac{1}{2}}} \sum_{m=0}^{n-4 k_{n}+1} \sum_{l=0}^{2 k_{n}-1} E\left[\hat{\chi}_{m, l}^{n}-\check{\chi}_{m, l}^{n} \mid \mathcal{F}_{\frac{i}{n}}\right] \stackrel{P}{\longrightarrow} 0 .
$$

For the first result we can refer to the proof of Theorem 1 in Podolskij and Vetter [18] once again, but this time we take a closer look on the proof than in Lemma 3. Observe that it suffices to show

$$
\begin{aligned}
& \frac{2}{n^{\frac{3}{2}}} \sum_{i=0}^{n-4 k_{n}+1} \sum_{j=0}^{2 k_{n}} n^{\frac{r_{1}+l_{1}}{2}}\left(E\left[\left|\bar{Z}_{i}^{\prime}{ }^{n}\right|^{l_{1}}\left|{\overline{Z^{\prime}}}_{i+k_{n}}^{n}\right|^{r_{1}}\left|{\overline{Z^{\prime}}}_{i+j}^{n}\right|^{l_{1}}\left|{\overline{Z^{\prime}}}_{i+k_{n}+j}^{n}\right|^{r_{1}} \mid \mathcal{F}_{\frac{i}{n}}\right]\right. \\
- & \left.E\left[\left|\bar{Y}_{i, i}^{n}\right|^{l_{1}}\left|\bar{Y}_{i+k_{n}, i}^{n}\right|^{r_{1}}\left|\bar{Y}_{i+j, i}^{n}\right|^{l_{1}}\left|\bar{Y}_{i+k_{n}+j, i}^{n}\right|^{r_{1}} \mid \mathcal{F}_{\frac{i}{n}}\right]\right) \stackrel{P}{\longrightarrow} 0 .
\end{aligned}
$$

in order to obtain (5.26). However, since

$$
\begin{aligned}
& \left|{\overline{Z^{\prime}}}_{i}^{n}\right|^{l_{1}}\left|{\overline{Z^{\prime}}}_{i+k_{n}}^{n}\right|^{r_{1}}\left|{\overline{Z^{\prime}}}_{i+j}^{n}\right|^{l_{1}}\left|{\overline{Z^{\prime}}}_{i+k_{n}+j}^{n}\right|^{r_{1}}-\left|\bar{Y}_{i, i}^{n}\right|^{l_{1}}\left|\bar{Y}_{i+k_{n}, i}^{n}\right|^{r_{1}}\left|\bar{Y}_{i+j, i}^{n}\right|^{l_{1}}\left|\bar{Y}_{i+k_{n}+j, i}^{n}\right|^{r_{1}} \\
= & \left(\left|{\overline{Z^{\prime}}}_{i}^{n}\right|^{l_{1}}-\left|\bar{Y}_{i, i}^{n}\right|^{l_{1}}\right)\left|{\overline{Z^{\prime}}}_{i+k_{n}}^{n}\right|^{r_{1}}\left|{\overline{Z^{\prime}}}_{i+j}^{n}\right|^{l_{1}}\left|{\overline{Z^{\prime}}}_{i+k_{n}+j}^{n}\right|^{r_{1}} \\
+ & \left|\bar{Y}_{i, i}^{n}\right|^{l_{1}}\left(\left|\bar{Z}_{i+k_{n}}^{n}\right|^{r_{1}}\left|{\overline{Z^{\prime}}}_{i+j}^{n}\right|^{l_{1}}\left|{\overline{Z^{\prime}}}_{i+k_{n}+j}^{n}\right|^{r_{1}}-\left|\bar{Y}_{i+k_{n}, i}^{n}\right|^{r_{1}}\left|\bar{Y}_{i+j, i}^{n}\right|^{l_{1}}\left|\bar{Y}_{i+k_{n}+j, i}^{n}\right|^{r_{1}}\right)
\end{aligned}
$$

we can conclude from Lemma 3 in Podolskij and Vetter [18] and through a recursive argument that (5.26) is proven, as long as

$$
\frac{2}{n^{\frac{3}{2}}} \sum_{i=0}^{n-4 k_{n}+1} \sum_{j=0}^{2 k_{n}} E\left[n^{\frac{1}{2}}\left({\overline{Z^{\prime}}}_{i}^{n}-\bar{Y}_{j, i}^{n}\right)^{2}\right] \stackrel{P}{\longrightarrow} 0 .
$$

However, this follows in the same manner as in Podolskij and Vetter [18].

We will establish (5.27) solely in model (1.3), which is enough to obtain the result in model (1.1) as well. Note that the claim reduces to the following two steps:

$$
\begin{aligned}
& \frac{2}{n^{\frac{3}{2}}} \sum_{i=0}^{n-4 k_{n}+1} \sum_{j=0}^{2 k_{n}} n^{\frac{r_{1}+l_{1}}{2}} E\left[\left(\left.\left|\bar{Z}_{i}^{\prime}\right|^{n}\right|^{l_{1}}\left|{\overline{Z^{\prime}}}_{i+k_{n}}^{n}\right|^{r_{1}}\left|{\overline{Z^{\prime}}}_{i+j}^{n}\right|^{l_{1}}\left|{\overline{Z^{\prime}}}_{i+k_{n}+j}^{n}\right|^{r_{1}}\right.\right. \\
- & \left.\left.\left|\bar{Z}_{i}^{n}\right|^{l_{1}}\left|\bar{Z}_{i+k_{n}}^{n}\right|^{r_{1}}\left|\bar{Z}_{i+j}^{n}\right|^{l_{1}}\left|\bar{Z}_{i+k_{n}+j}^{n}\right|^{r_{1}}\right) 1_{A_{i, j}^{c}} \mid \mathcal{F}_{\frac{i}{n}}\right] \stackrel{P}{\longrightarrow} 0
\end{aligned}
$$


and

$$
\frac{2}{n^{\frac{3}{2}}} \sum_{i=0}^{n-4 k_{n}+1} \sum_{j=0}^{2 k_{n}} n^{\frac{r_{1}+l_{1}}{2}} E\left[\left|{\overline{Z^{\prime}}}_{i}^{n}\right|^{l_{1}}\left|{\overline{Z^{\prime}}}_{i+k_{n}}^{n}\right|^{r_{1}}\left|{\overline{Z^{\prime}}}_{i+j}^{n}\right|^{l_{1}}\left|{\overline{Z^{\prime}}}_{i+k_{n}+j}^{n}\right|^{r_{1}} 1_{A_{i, j}} \mid \mathcal{F}_{\frac{i}{n}}\right] \stackrel{P}{\longrightarrow} 0
$$

where $A_{i, j}$ is the set on which at least one of the random variables $\left|\bar{Z}_{m}^{n}\right|$ with $m=i, i+k_{n}, i+j, i+k_{n}+j$ is larger than $n^{-\varpi}$. Since

$$
1_{\left\{\left|\bar{Z}_{m}^{n}\right| \geq n^{-\varpi}\right\}} \leq 1_{\left\{\left|\bar{Z}_{m}^{\prime}\right| \geq \frac{n^{-\varpi}}{2}\right\}}+1_{\left\{\left|{\overline{Z^{\prime \prime}}}_{m}^{n}\right| \geq \frac{n^{-\varpi}}{2}\right\}}
$$

the second result is an easy application of Markov's and Hölder's inequality, due to

$$
E\left[\mid{\overline{Z^{\prime}}}_{i}^{n} \| \mathcal{F}_{\frac{i}{n}}\right] \leq C n^{-\frac{1}{4}} \quad \text { and } \quad E\left[\mid{\overline{Z^{\prime \prime}}}_{i}^{n} \| \mathcal{F}_{\frac{i}{n}}\right] \leq C n^{-\frac{1}{4}}
$$

and the moment assumption on $U$.

The proof of the first claim is more involved. Note first that from a similar argument as in (5.28) one can deduce the result from

$$
\frac{2}{n^{\frac{3}{2}}} \sum_{i=0}^{n-4 k_{n}+1} \sum_{j=0}^{2 k_{n}} n^{\frac{r_{1}+l_{1}}{2}} E\left[\left|{\overline{Z^{\prime \prime}}}_{i}^{n}\right|^{l_{1}}\left|{\overline{Z^{\prime \prime}}}_{i+k_{n}}^{n}\right|^{r_{1}}\left|{\overline{Z^{\prime \prime}}}_{i+j}^{n}\right|^{l_{1}}\left|{\overline{Z^{\prime \prime}}}_{i+k_{n}+j}^{n}\right|^{r_{1}} 1_{A_{i, j}^{c}} \mid \mathcal{F}_{\frac{i}{n}}\right] \stackrel{P}{\longrightarrow} 0
$$

which can easily be reduced to the proof of

$$
n^{\frac{l_{1}}{4}} E\left[\left|{\overline{Z^{\prime \prime}}}_{i}^{n}\right|^{q} 1_{\left\{\left|\bar{Z}_{i}^{n}\right|<n^{-\varpi}\right\}} \mid \mathcal{F}_{\frac{i}{n}}\right]^{\frac{1}{\delta}} \stackrel{P}{\longrightarrow} 0
$$

for some $q=l_{1} \cdot \delta$ with $\delta>1$ small enough and uniformly in $i$. Since

$$
1_{\left\{\left|\bar{Z}_{i}^{n}\right|<n^{-\varpi}\right\}} \leq 1_{\left\{\left|{\overline{Z^{\prime \prime}}}_{i}^{n}\right|<2 n^{-\varpi}\right\}}+1_{\left\{\left|{\overline{Z^{\prime \prime}}}_{i}^{n}\right| \geq 2 n^{-\varpi}\right\}} 1_{\left\{\left|{\overline{Z^{\prime}}}_{i}^{n}\right| \geq n^{-\varpi}\right\}},
$$

the claim can further be reduced to

$$
n^{\frac{l_{1}}{4}} E\left[\left|{\overline{Z^{\prime \prime}}}_{i}^{n}\right|^{q} 1_{\left\{\left|{\overline{Z^{\prime}}}_{i}^{n}\right| \geq n^{-\varpi}\right\}} \mid \mathcal{F}_{\frac{i}{n}}\right]^{\frac{1}{\delta}} \stackrel{P}{\longrightarrow} 0
$$

uniformly in $i$. By means of Hölder's inequality and for some $p$ large enough we obtain

$$
E\left[\left|{\overline{Z^{\prime \prime}}}_{i}^{n}\right|^{q} 1_{\left\{\left|{\overline{Z^{\prime}}}_{i}^{n}\right| \geq n^{-\varpi}\right\}} \mid \mathcal{F}_{\frac{i}{n}}\right]^{\frac{1}{\delta}} \leq E\left[\left|{\overline{Z^{\prime \prime}}}_{i}^{n}\right|^{q p} \mid \mathcal{F}_{\frac{i}{n}}{ }^{\frac{1}{p \delta}} P\left(\left|\bar{Z}_{i}^{n}\right| \geq n^{-\varpi} \mid \mathcal{F}_{\frac{i}{n}}\right)^{\frac{p-1}{p \delta}}\right.
$$

Since the first term is bounded (uniformly in $i$ ), but not necessarily of order $n^{-\frac{l_{1}}{4}}$ as for $q p \leq 2$, we see that we are left to prove that

$$
P\left(\left|{\overline{Z^{\prime}}}_{i}^{n}\right| \geq n^{-\varpi} \mid \mathcal{F}_{\frac{i}{n}}\right)=o\left(n^{-\frac{p q}{4(p-1)}}\right),
$$


uniformly in $i$. From

$$
\left|{\overline{Z^{\prime}}}_{i}^{n}\right| \leq\left|\bar{Q}_{i}^{n}\right|+\left|\bar{U}_{i}^{n}\right| \leq C\left|\overline{\Delta_{i}^{n} W}\right|+\left|\bar{U}_{i}^{n}\right|
$$

the claim can further be reduced to

$$
P\left(\left|\bar{U}_{i}^{n}\right| \geq n^{-\varpi}\right)=o\left(n^{-\frac{q p}{4(p-1)}}\right) \quad \text { and } \quad P\left(\left|\overline{\Delta_{i}^{n} W}\right| \geq n^{-\varpi}\right)=o\left(n^{-\frac{q p}{4(p-1)}}\right) .
$$

Both results follow easily form Markov's inequality. For the first one we have

$$
P\left(\left|\bar{U}_{i}^{n}\right| \geq n^{-\varpi}\right)=P\left(\left|n^{\frac{1}{4}} \bar{U}_{i}^{n}\right| \geq n^{\frac{1}{4}-\varpi}\right)
$$

Thus for some $t>\frac{q p}{4(p-1)\left(\frac{1}{4}-\varpi\right)}$ and some $\eta>0$ we obtain

$$
P\left(\left|\bar{U}_{i}^{n}\right| \geq n^{-\varpi}\right) \leq \frac{E\left[\left|n^{\frac{1}{4}} \bar{U}_{i}^{n}\right|^{t}\right]}{n^{t\left(\frac{1}{4}-\varpi\right)}} \leq C n^{-\left(\frac{q p}{4(p-1)}+\eta\right)},
$$

since the $t$-th moment of $U$ is finite by assumption. The result for the Brownian part follows in the same way.

\section{Proof of Theorem 4}

We start with some results that can easily be concluded from condition (L- $q), q<1$. Recall that it is sufficient to replace the family of functions $\gamma_{k}$ by a bounded function $\gamma$. Note then that (L- $q)$ implies

$$
\int_{\mathbb{R}}|\gamma(x)|^{r} d x<\infty
$$

for all $q \leq r<1$, since with $A_{0}=\{|\gamma(x)| \leq 1\}$ and $A_{1}=\{|\gamma(x)|>1\}$ we have

$$
\int_{A_{0}}|\gamma(x)|^{q} d x<\infty \quad \text { and } \quad \lambda\left(A_{1}\right)<\infty,
$$

where $\lambda$ denotes the Lebesgue measure. Therefore

$$
\int_{A_{0}}|\gamma(x)|^{r} d x \leq \int_{A_{0}}|\gamma(x)|^{q} d x<\infty
$$

and

$$
\int_{A_{1}}|\gamma(x)|^{r} d x \leq C^{r} \lambda\left(A_{1}\right)<\infty
$$

since $\gamma$ is assumed to be bounded. 
Let further $v(X)_{t}$ denote the variation process of some process $X$ up to time $t$. By assumption, $\kappa$ is a truncation function with bounded support, from which we can conclude that

$$
\kappa(x) \leq C 1_{\{|x| \leq b\}}(x)
$$

for some constants $b$ and $C$. We see easily that $\kappa \star \nu_{t}$ is of finite variation for any $t$, since due to condition $(\mathrm{H})$

$$
\begin{aligned}
& v(\kappa \star \nu)_{t} \leq \int_{0}^{t}\left|\int_{\mathbb{R}} \kappa \circ \delta(s, x) d x\right| d s \\
\leq & \int_{0}^{t} \int_{\mathbb{R}}|\delta(s, x)| 1_{\{|\delta(s, x)| \leq 1\}} d x d s+\int_{0}^{t} \int_{\mathbb{R}}|\delta(s, x)| 1_{\{1<|\delta(s, x)| \leq b\}} d x d s .
\end{aligned}
$$

For the first integral we have

$$
\begin{aligned}
\int_{0}^{t} \int_{\mathbb{R}}|\delta(s, x)| 1_{\{|\delta(s, x)| \leq 1\}} d x d s & \leq \int_{0}^{t} \int_{\mathbb{R}}(|\delta(s, x)| \wedge 1) d x d s \\
& \leq \int_{0}^{t} \int_{\mathbb{R}} \Phi_{1}(\gamma(x)) d x d s \\
& \leq t \int_{\mathbb{R}} \Phi_{q}(\gamma(x)) d x<\infty .
\end{aligned}
$$

The latter one satisfies

$$
\begin{aligned}
\int_{0}^{t} \int_{\mathbb{R}}|\delta(s, x)| 1_{\{1<|\delta(s, x)| \leq b\}} d x d s & \leq \int_{0}^{t} \int_{\mathbb{R}}|\gamma(x)| 1_{\{|\gamma(x)|>1\}} d x d s \\
& =t \int_{A_{1}}|\gamma(x)| d x<\infty
\end{aligned}
$$

for the same reason as above. Therefore, $X$ can be decomposed as

$$
X_{t}=X_{0}+\bar{B}_{t}+Q_{t}+\sum_{s \leq t} \Delta X_{s},
$$

with $\bar{B}_{t}=B_{t}-\kappa \star \nu_{t}$ being of finite variation. $Q_{t}$ denotes the continuous martingale part of $X$ as in (5.3).

Let us now come to the proof of Theorem 4. It is easy to see that $\bar{B}_{t}$ inherits all properties of a typical drift process. Therefore, we know that the assertion from Theorem 3 holds for the process

$$
Z_{t}^{\prime \prime \prime}=X_{0}+\bar{B}_{t}+Q_{t}+U_{t} .
$$


It remains to show

$$
n^{\frac{1}{4}} E\left[\left|B T(Z, l, r)^{n}-B T\left(Z^{\prime \prime \prime}, l, r\right)^{n}\right|\right] \stackrel{P}{\longrightarrow} 0
$$

which similarly to the proof of Theorem 2 can be reduced to

$$
\sup _{i} n^{\frac{p+1}{4}} E\left[\left.|| \bar{Z}_{i}^{n}\right|^{p}-\left|{\overline{Z_{i}^{\prime \prime \prime}}}_{i}^{n}\right|^{p}|| \mathcal{F}_{\frac{i}{n}}\right] \leq \alpha_{n}
$$

for all $\frac{q}{2-q}<p<1$ and some $\alpha_{n} \rightarrow 0$. From ||$x+\left.y\right|^{p}-\left.|x|^{p}|\leq| y\right|^{p}$ for $p \leq 1$ we conclude that it is sufficient to prove

$$
\sup _{i} n^{\frac{p+1}{4}} E\left[\left|\bar{J}_{i}^{n}\right|^{p} \mid \mathcal{F}_{\frac{i}{n}}\right] \leq \alpha_{n}
$$

with $J_{t}=\sum_{s \leq t} \Delta X_{s}$. But

$$
\begin{aligned}
E\left[\left|\bar{J}_{i}^{n}\right|^{p} \mid \mathcal{F}_{\frac{i}{n}}\right] & \leq C E\left[\left|\overline{\Delta_{i}^{n} J}\right|^{p} \mid \mathcal{F}_{\frac{i}{n}}\right] \leq C E\left[\sum_{\frac{i}{n}<s \leq \frac{i+k_{n}}{n}}\left|\Delta X_{s}\right|^{p} \mid \mathcal{F}_{\frac{i}{n}}\right] \\
& \leq C \int_{\frac{i}{n}}^{\frac{i+k_{n}}{n}} \int_{\mathbb{R}}|\delta(s, x)|^{p} d s d x \leq C \int_{\frac{i}{n}}^{\frac{i+k_{n}}{n}} \int_{\mathbb{R}}|\gamma(x)|^{p} d s d x \\
& \leq C n^{-\frac{1}{2}},
\end{aligned}
$$

whenever $p \geq q$. (5.29) is then equivalent to $q \leq p<1$. On the other hand, for $q>p$ we conclude from Hölder's inequality that

$$
E\left[\left|\bar{J}_{i}^{n}\right|^{p} \mid \mathcal{F}_{\frac{i}{n}}\right] \leq E\left[\left|\bar{J}_{i}^{n}\right|^{q} \mid \mathcal{F}_{\frac{i}{n}}\right]^{\frac{p}{q}} \leq C n^{-\frac{p}{2 q}}
$$

Therefore (5.29) holds in this case, provided $\frac{q}{2-q}<p<q$. We conclude that (5.29) holds, as long as $\frac{q}{2-q}<p<1$. This proves the result.

\section{References}

[1] Y. Ait-Sahalia, J. Jacod (2008). Testing for jumps in a discretely observed process. Ann. Stat. (forthcoming)

[2] D. J. Aldous, G. K. Eagleson (1978). On mixing and stability of limit theorems. Ann. Probab. 6 (2), 325-331.

[3] T. G. Andersen, T. Bollerslev, F. X. Diebold, P. Labys (2001). The distribution of exchange rate volatility. J. Amer. Statist. Assoc. 96, 42-55. 
[4] O. E. Barndorff-Nielsen, S. E. Graversen, J. Jacod, M. Podolskij, N. Shephard (2006). A central limit theorem for realised power and bipower variations of continuous semimartingales. In Y. Kabanov, R. Lipster and J. Stoyanov (eds.), From Stochastic Calculus to Mathematical Finance: The Shiryaev Festschrift, 33-68. Berlin, Springer-Verlag.

[5] O. E. Barndorff-Nielsen, P. R. Hansen, A. Lunde, N. Shephard (2006). Designing realised kernels to measure the ex-post variation of equity prices in the presence of noise. Econometrica (forthcoming).

[6] O. E. Barndorff-Nielsen, N. Shephard (2002). Econometric analysis of realised volatility and its use in estimating stochastic volatility models. J. Roy. Statist. Soc. Ser. B 64, 253-280.

[7] O. E. Barndorff-Nielsen, N. Shephard (2004). Power and bipower variation with stochastic volatility and jumps. J. Financial Econometrics 2, 1-48.

[8] F. Black, M. Scholes (1973). The pricing of options and corporate liabilities. J. Polit. Econ. 81, 637-654.

[9] K. C. Chan, A. G. Karolyi, F. A. Longstaff, A. B. Sanders (1992). An empirical comparison of alternative models of the short-term interest rate. J. Finance 47, 1209-1227.

[10] J. C. Cox, J. E. Ingersoll, S. A. Ross (1980). An analysis of variable rate loan contracts. J. Finance 35, 389-403.

[11] A. Gloter, J. Jacod (2001). Diffusions with measurement errors. II. Optimal estimators. ESAIM Probability and Statistics 5, 243-260.

[12] J. Jacod (2008). Asymptotic properties of realized power variations and related functionals of semimartingales. Stochastic Process. Appl. 118 (4), 517-559.

[13] J. Jacod (2006). Asymptotic properties of realized power variations and related functionals of semimartingales: Multipower variations. Tech. Rep., Université de Paris VI.

[14] J. Jacod, Y. Li, P. A. Mykland, M. Podolskij, M. Vetter (2007). Microstructure Noise in the Continuous Case: The Pre-Averaging Approach. Tech. Rep., RuhrUniversität Bochum. 
[15] J. Jacod, M. Podolskij, M. Vetter (2008). Limit theorems for moving averages of discretized processes plus noise. Tech. Rep., Université de Paris VI.

[16] J. Jacod, A. N. Shiryaev (2003). Limit theorems for stochastic processes. Berlin, Springer-Verlag.

[17] S. N. Lahiri (2003). Resampling methods for dependent data. Springer, New York.

[18] M. Podolskij, M. Vetter (2006). Estimation of Volatility Functionals in the Simultaneous Presence of Microstructure Noise and Jumps. Tech. Rep., RuhrUniversität Bochum.

[19] A. Renyi (1963). On stable sequences of events. Sankhya Ser. A 25, 293-302.

[20] O. A. Vasicek (1977). An equilibrium characterization of the term structure. J. Financial Economics 5, 177-188.

[21] L. Zhang (2004). Efficient estimation of stochastic volatility using noisy observations: a multiscale approach. Bernoulli 12 (6), 1019-1043.

[22] L. Zhang, P. A. Mykland, Y. Ait-Sahalia (2005). A tale of two time scales: Determining integrated volatility with noisy high-frequency data. J. Amer. Statist. Assoc. 472, 1394-1411. 